\title{
Saliva from nymph and adult females of Haemaphysalis longicornis: a proteomic study
}

Lucas Tirloni ${ }^{1}$, Mohammad Saiful Islam, ${ }^{2,3}$, Tae Kwon Kim ${ }^{6}$, Jolene K. Diedrich ${ }^{4}$, John R. Yates $\|^{4}{ }^{4}$, Antônio F. M. Pinto ${ }^{4,5}$, Albert Mulenga ${ }^{6}$, Myung-Jo You ${ }^{2}$ and Itabajara Da Silva Vaz Jr. ${ }^{1,7^{*}}$

\begin{abstract}
Background: Haemaphysalis longicornis is a major vector of Theileria spp., Anaplasma phagocytophilum, Babesia spp. and Coxiella burnetti in East Asian countries. All life stages of ixodid ticks have a destructive pool-feeding style in which they create a pool-feeding site by lacerating host tissue and secreting a variety of biologically active compounds that allows the tick to evade host responses, enabling the uptake of a blood meal. The identification and functional characterization of tick saliva proteins can be useful to elucidate the molecular mechanisms involved in tick development and to conceive new anti-tick control methods.
\end{abstract}

Methods: H. longicornis tick saliva was collected from fully engorged nymphs and fully engorged adults induced by dopamine or pilocarpine, respectively. Saliva was digested with trypsin for LC-MS/MS sequencing and peptides were searched against tick and rabbit sequences.

Results: A total of 275 proteins were identified, of which 135 were tick and 100 were rabbit proteins. Of the tick proteins, 30 proteins were identified exclusively in fully engorged nymph saliva, 74 in fully engorged adult females, and 31 were detected in both stages. The identified tick proteins include heme/iron metabolism-related proteins, oxidation/detoxification proteins, enzymes, proteinase inhibitors, tick-specific protein families, and cytoskeletal proteins. Proteins involved in signal transduction, transport and metabolism of carbohydrate, energy, nucleotide, amino acids and lipids were also detected. Of the rabbit proteins, 13 were present in nymph saliva, 48 in adult saliva, and 30 were present in both. The host proteins include immunoglobulins, complement system proteins, antimicrobial proteins, serum albumin, peroxiredoxin, serotransferrin, apolipoprotein, hemopexin, proteinase inhibitors, and hemoglobin/red blood cells-related products.

Conclusions: This study allows the identification of $\mathrm{H}$. longicornis saliva proteins. In spontaneously detached tick saliva various proteins were identified, although results obtained with saliva of fully engorged ticks need to be carefully interpreted. However, it is interesting to note that proteins identified in this study were also described in other tick saliva proteomes using partially engorged tick saliva, including hemelipoprotein, proteases, protease inhibitors, proteins related to structural functions, transporter activity, metabolic processes, and others. In conclusion, these data can provide a deeper understanding to the biology of H. longicornis.

Keywords: Tick, Proteomic, Saliva, Tick-host relationship

\footnotetext{
* Correspondence: itabajara.vaz@ufrgs.br

${ }^{1}$ Centro de Biotecnologia, Universidade Federal do Rio Grande do Sul, Porto

Alegre, RS, Brazil

${ }^{7}$ Faculdade de Veterinária, Universidade Federal do Rio Grande do Sul, Porto

Alegre, RS, Brazil

Full list of author information is available at the end of the article
}

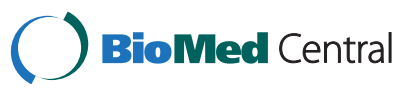

(c) 2015 Tirloni et al. This is an Open Access article distributed under the terms of the Creative Commons Attribution License (http://creativecommons.org/licenses/by/4.0), which permits unrestricted use, distribution, and reproduction in any medium, provided the original work is properly credited. The Creative Commons Public Domain Dedication waiver (http:// creativecommons.org/publicdomain/zero/1.0/) applies to the data made available in this article, unless otherwise stated. 


\section{Background}

The hard tick Haemaphysalis longicornis is a medically and veterinary important vector of many tick-borne disease (TBD), transmitting pathogens such as Ehrlichia chaffeensis [1], Anaplasma bovis [2], A. phagocytophilum [3], Coxiella burnetii [4], and Spotted fever group rickettsiae [5]. Of significant veterinary importance, this tick species is considered the primary vector of theileriosis caused by Theileria spp. and of babesiosis caused by Babesia spp. in both sheep and cattle in East Asia $[6,7]$. As a three-host tick, it has a wide range of hosts, from birds and lagomorphs (as immature ticks), and large domestic and wild mammals (as adult parasites). This tick is vastly distributed throughout Eastern Asian countries such as China, Korea, Japan, New Zealand, and Australia [8].

The tick feeding process is initiated when the tick engages and attaches onto its host. As a pool feeder, upon attachment the tick determines a suitable feeding site, and prepares it by lacerating small blood vessels. Feeding occurs by sucking up the blood that flows to the pool. This feeding style triggers host defense mechanisms such as pain or itching, hemostasis, inflammatory reactions, tissue repair, and immune rejection [9-12]. To control the feeding site and counteract the host defenses, ticks secrete and inject saliva into its host, of which contains hundreds of different proteins [7, 13-16] and other pharmacologically active molecules that confer antihemostatic, anti-inflammatory and immunomodulatory properties, supporting blood feeding [17-20].

During the feeding process, infected ticks may transmit TBD-causing pathogens. Besides being a critical component of the feeding process, saliva has also been shown to play a role in pathogen transmission [21]. Therefore, the identification and characterization of novel $H$. longicornis saliva proteins could point to candidates for the development of anti-tick and transmissionblocking vaccines [22-26] and of new pharmacological active molecules for medical application [18, 20, 27-29].

Currently the saliva proteome of Amblyomma americanum, Ixodes scapularis, Ornithodoros moubata, Rhipicephalus sanguineus sensu lato, $R$. microplus, and Dermacentor andersoni [13-16, 30-33] tick species have been analyzed. However, $H$. longicornis tick saliva proteome has not been the object of any analysis. The objectives of this study were to identify secreted proteins in the saliva of fully engorged nymphs (nymphs) and fully engorged adult females (adults) of $H$. longicornis ticks, comparing the protein profile of these developmental tick stages to evaluate the variation in tick saliva during feeding of different life stages. This affords to identify tick saliva proteins shared by the two developmental stages. Such proteins may play an important role in the success of both developmental stages in their feeding cycle. The novel catalog of tick saliva proteins identified in this study provides a deeper understanding to the biology of $H$. longicornis.

\section{Methods}

\section{Ethics statement ethical approval}

All animals used in these experiments were housed in Laboratory of Veterinary Parasitology, College of Veterinary Medicine and Bio-Safety Research Institute in Chonbuk National University, Jeonju 561-756, Republic of Korea. All animal studies and protocols are in agreement with the ethical principles for animal research and approved by the Chonbuk Animal Care and Use Committee (CBNU 2015-003).

\section{Ticks and saliva collection}

The Jeju strain of the hard tick $H$. longicornis has been maintained on rabbits in our laboratory since 2003. To feed, $H$. longicornis ticks were placed onto the ears of specific pathogen-free (SPF) New Zealand White rabbits. Ticks were restricted to the ear using cloth pocket-like socks attached on ear ending with tape. Approximately 150 nymphs and 150 adults were placed in their respective feeding apparatuses and allowed to feed upon full engorgement and spontaneous detachment. Saliva was collected from 90 nymphs and 50 adults that were fully engorged and that detached from the rabbits spontaneously. Ticks were rinsed with sterile distilled water and induced to salivate by dorsal injection (posterior to fourth coxae in the region of epimeral and anal plates) of 5.0 to $7.0 \mu \mathrm{L} 0.2 \%$ dopamine or 1.5 to $3.0 \mu \mathrm{l} 2 \%$ pilocarpine (in $10 \mathrm{mM}$ Tris-EDTA buffer) for nymphs and adults, respectively. Injections were applied using a micro-fine especially homemade glass needle. Then, ticks were maintained at $37{ }^{\circ} \mathrm{C}$ in an $85 \%$ relative humidity chamber, and saliva was periodically collected for approximately $4 \mathrm{~h}$ using a pipette tip. Tick saliva was lyophilized and stored at $-80{ }^{\circ} \mathrm{C}$ for LC-MS/MS analysis. Saliva protein concentrations were determined by Qubitfluorometer (Life Technologies, Carlsbad, CA, USA).

\section{Protein digestion and sample preparation}

Saliva of $H$. longicornis nymphs and adult females was digested in solution with trypsin. Lyophilized salivary proteins were dissolved in $8 \mathrm{M}$ urea/0.1 M Tris, $\mathrm{pH} 8.5$, reduced with $5 \mathrm{mMTris}$ (2-carboxyethyl)phosphine hydrochloride (TCEP, Sigma-Aldrich, St Louis, MO, USA) and alkylated with $25 \mathrm{mM}$ iodoaceamide (Sigma-Aldrich). Proteins were digested overnight at $37{ }^{\circ} \mathrm{C}$ in $2 \mathrm{M}$ urea/0.1 M Tris $\mathrm{pH} 8.5,1 \mathrm{mM} \mathrm{CaCl}_{2}$ with trypsin (Promega, Madison, WI, USA) at a final 1:20 ratio (enzyme:substrate). Digestions were stopped with formic acid (5 \% final concentration) and centrifuged for debris removal. 


\section{Precolumns and analytical columns}

Reversed phase pre-columns were prepared by first creating a Kasil frit at one end of a deactivated 250- $\mu \mathrm{m} \mathrm{ID/}$ 360- $\mu \mathrm{m}$ OD capillary (Agilent Technologies, Santa Clara, CA, USA). Kasil frits were prepared by dipping $20 \mathrm{~cm}$ capillary in $300 \mu \mathrm{L}$ Kasil 1624 (PQ Corporation, Malvern, PA, USA) and $100 \mu \mathrm{L}$ formamide solution, curing at $100{ }^{\circ} \mathrm{C}$ for $3 \mathrm{~h}$, and cutting the frit to a length of $2 \mathrm{~mm}$. Precolumns were packed in-house with $5 \mu \mathrm{m}$ ODS-AQ C18 (YMC America, INC., Allentown, PA, USA) particles from particle slurries in methanol upon reaching the height of $2 \mathrm{~cm}$. Analytical reversed phase columns were assembled by pulling a $100-\mu \mathrm{m}$ ID/360- $\mu \mathrm{m}$ OD (Molex Polymicro Technologies $^{\mathrm{ma}}$, Austin, TX, USA) silica capillary to a $5 \mu \mathrm{m}$ ID tip. The same packing material was packed directly into the pulled column until the length of $20 \mathrm{~cm}$ was reached. Reversed phase precolumns and analytical columns were connected using a zero-dead volume union (IDEX Corp., Upchurch Scientific, Oak Harbor, WA, USA).

\section{LC-MS/MS}

Peptide mixtures were analyzed by nanoflow liquid chromatography mass spectrometry using an Easy NanoLC II and a Q Exactive mass spectrometer (Thermo Scientific, Waltham, MA, USA). Peptides eluted from the analytical column were electrosprayed directly into the mass spectrometer. Buffer A and B consisted of $5 \%$ acetonitrile $/ 0.1 \%$ formic acid and $80 \%$ acetonitrile/ $0.1 \%$ formic acid, respectively. The flow rate was set to $400 \mathrm{~nL} /$ min. H. longicornis digested saliva samples $(1.5 \mu \mathrm{g}$ per injection) were separated in $155 \mathrm{~min}$ chromatographic runs, with linear gradient from 1 to $10 \%$ of buffer B for $10 \mathrm{~min}$ followed by an increase to $40 \%$ of buffer B in $100 \mathrm{~min}$, an increase to $50 \%$ of buffer B in $10 \mathrm{~min}$ and finally an increase to $90 \%$ of buffer B for additional 10 min. Column was held at $90 \%$ of buffer B for $10 \mathrm{~min}$, reduced to $1 \%$ of buffer $\mathrm{B}$ and re-equilibrated prior to the next injection.

The mass spectrometer was operated in a data dependent mode, collecting a full MS scan from 400 to $1200 \mathrm{~m} / \mathrm{z}$ at 70,000 resolution and an AGC target of $1 \times 10^{6}$. The 10 most abundant ions per scan were selected for MS/MS at 17,500 resolution and AGC target of $2 \times 10^{5}$ and an underfill ratio of $0.1 \%$. Maximum fill times were 20 and $120 \mathrm{~ms}$ for MS and MS/MS scans, respectively, with dynamic exclusion of $15 \mathrm{~s}$. Normalized collision energy was set to 25 .

\section{Data analysis}

Tandem mass spectra were extracted from raw files using RawExtract 1.9.9.2 [34] and searched with ProLuCID [35] against a combined non-redundant database containing (i) Ixodidae database from NCBI; (ii) R. microplus transcriptome database (Rm-INCT-EM, containing
22,009 sequences produced by our research group using Illumina Sequencing technology - BioProject ID PRJNA 232001 at Transcriptome Shotgun Assembly (TSA) database - GenBank); (iii) Oryctolagus cuniculus database from SwissProt and (iv) reverse sequences of all database entries. Searches were done using an Integrated Proteomics Pipeline - IP2 (Integrated Proteomics Applications) for The Scripps Institute (La Jolla, CA, USA). The search space included all fully-tryptic and half-tryptic peptide candidates. Carbamidomethylation on cysteine was used as static modification. Data was searched with 50-ppm precursor ion tolerance and 20-ppm fragment ion tolerance.

The validity of the peptide spectrum matches (PSMs) generated by ProLuCID was assessed using Search Engine Processor (SEPro) [36]. Identifications were grouped by charge state and tryptic status, resulting in four distinct subgroups. For each group, ProLuCIDXCorr, DeltaCN, DeltaMass, ZScore, number of peaks matched and Spec Count Score values were used to generate a Bayesian discriminator. The identifications were sorted in a nondecreasing order according to the discriminator score. A cutoff score was established to accept a false discovery rate (FDR) of $1 \%$ based on the number of decoys. This procedure was independently performed on each data subset, resulting in a false-positive rate that was independent of tryptic status or charge state. Additionally, a minimum sequence length of six residues per peptide was required. Results were post-processed to only accept PSMs with < $10 \mathrm{ppm}$ precursor mass error.

A Volcano plot was generated by a pairwise comparison between nymphs and fully engorged female $H$. longicornis tick saliva using the TFold module from PatternLab for Proteomics platform [37]. The following parameters were used to select differentially expressed proteins: proteins were grouped by maximum parsimony, spectral count data was normalized using normalized spectral abundance factor (NSAF) [38], and two nonzero replicate values were required for each condition (at least two out of four replicates). A BH q-value was set at 0.02 ( $2 \%$ FDR). A variable fold-change cutoff for each individual protein was calculated according to the $t$-test $p$-value using an F-Stringency value automatically optimized by the TFold software. Low abundant proteins were removed using an L-stringency value of 0.4 .

Venn diagrams were manually generated from the output of PatternLab's Birds Eye view report. Proteins were grouped by maximum parsimony and the presence of proteins in at least two out of four replicates was required for each condition.

\section{Functional annotation and classification}

To gain insight on the nature of the identified protein sequences, BLASTp searches against several databases 
were performed. To check the identity of tick saliva proteins detected, several databases were used for screening: non-redundant (NR), Acari and refseq-invertebrate from NCBI; Acari from Swissprot; the GeneOntology (GO) FASTA subset [39]; MEROPS database; and the conserved domains database of NCBI [40] containing the KOG [41], PFAM [42], and SMART motifs [43]. To check rabbit proteins identity, we used Oryctolagus cuniculus and refseq-vertebrates databases from NCBI; the conserved domains database of NCBI [40] containing the KOG [41], PFAM [42], and SMART motifs [43]; and the GeneOntology (GO) FASTA subset [39]. To functionally classify the protein sequences, a program written and provided by Dr. José M. C. Ribeiro in Visual Basic 6.0 (Microsoft, Redmond, Washington, USA) was used [44]. The functionally annotated catalog for each dataset was manually curated and plotted in a hyperlinked Excel spreadsheet (Additional file 1: Table S1 and Additional file 2: Table S2).

\section{Results and discussion}

$H$. longicornis is the primary tick known to transmit disease pathogens to humans and animals in East Asian countries [45]. Like several other blood-sucking parasites, its saliva secretion is a mixture of proteins produced in different salivary gland acinar cells [46]. Tick salivation can be studied in vivo by injection of dopamine, a neurotransmitter that stimulates fluid secretion by salivary gland, or by using pilocarpine, a cholinomimetic agent that induces the release of dopamine from the salivary nerves, resulting in salivation [47-49]. In Ornithodoros moubata, comparative analysis by SDSPAGE of several pilocarpine- and dopamine-induced saliva batches demonstrated reproducibility between both protocols used to induce tick salivation [13]. We have tested the use of pilocarpine and/or dopamine in both adult and nymph saliva collection. However, using pilocarpine for nymph saliva collection did not result in a substantial amount of saliva. When dopamine was used in adult ticks, the saliva acquired a darker color (data not shown). This observation using dopamine for adult saliva stimulation is in accordance with results described for $R$. sanguineus s.l. [15]. However, differently from data obtained for $R$. sanguineus s.l. adult tick saliva, we successfully collected and identified proteins from $H$. longicornis nymph saliva induced by dopamine. These differences could be associated to stage and/or species of ticks used in the salivation induction. Few tick saliva proteomes have been published, most of which using adult ticks and, therefore, knowledge about nymph saliva collection is not available.

At the time study was carried out, $H$. longicornis genome and transcriptome sequences were not available, therefore we screened against available Ixodidae databases from NCBI and an in-house $R$. microplus transcriptome database to identify tick salivary proteins by shotgun proteomics. Additionally, saliva was screened for host proteins using available rabbit protein sequences from Uniprot. The comparison in proteomic content between samples from different life cycle stages may show unique or increased levels of particular proteins that are important for tick biology. Moreover, it is possible to provide information about semi-quantitative variations on the levels of the specific proteins. Comparative proteomics of spontaneously detached fully engorged nymph and fully engorged adult females $H$. longicornis have shown alterations in protein salivary content through different life cycle stages, generating new insights into tick physiology. The main objective in this study was to evaluate the $H$. longicornis saliva proteome, showing saliva protein content in nymphs, as an immature stage, and in fully engorged adult females, as an experimental end-point, evaluating not only differences, but also the similarities in saliva contents at different stages of tick development. It is important to observe that analyses of saliva of fully engorged ticks have to be carefully interpreted, since they are at the end of the feeding process. However, this analysis can provide useful information, since various categories of proteins identified in this study were also described in other tick saliva proteomes using partially engorged tick saliva [13-16], including: proteins related to heme/iron metabolism (hemelipoprotein, ferritin), proteases (cathepsin, trypsin-like, metalloprotease), protease inhibitors (serpin, cystatin, alpha-2-macroglobulin, TIL), proteins related to structural functions, transporter activity, metabolic processes, protein modification machinery (heat shock proteins), and others. In addition, other proteins from fully engorged ticks have been characterized as anticoagulant molecules, like microphilin [27], BmAP [28], and haemalin [29]. Therefore, despite the limitation in using fully engorged adult saliva, the biological interpretation of these data can provide a deeper understanding to the biology of H. longicornis.

\section{An overview of identified saliva proteins}

Tick saliva was obtained by dopamine and pilocarpine stimulations from $H$. longicornis nymphs and adult females, respectively. The saliva accumulated in the mouthparts was periodically collected from the ticks using a pipette tip (Fig. 1a and b). Crystal particles formed around the nymph mouthpart were also collected (Fig. 1c). Collected saliva was subjected to tryptic digestion and analyzed by shotgun proteomics in quadruplicate. A total of 135 proteins were identified matching tick databases, and 100 proteins matching rabbit database (Fig. 2 and Tables 1 , 2 and 3). The identified tick proteins were classified and divided into groups according to their putative functions (Fig. 3 and Tables 1, 2 and 3), consistent with previously 

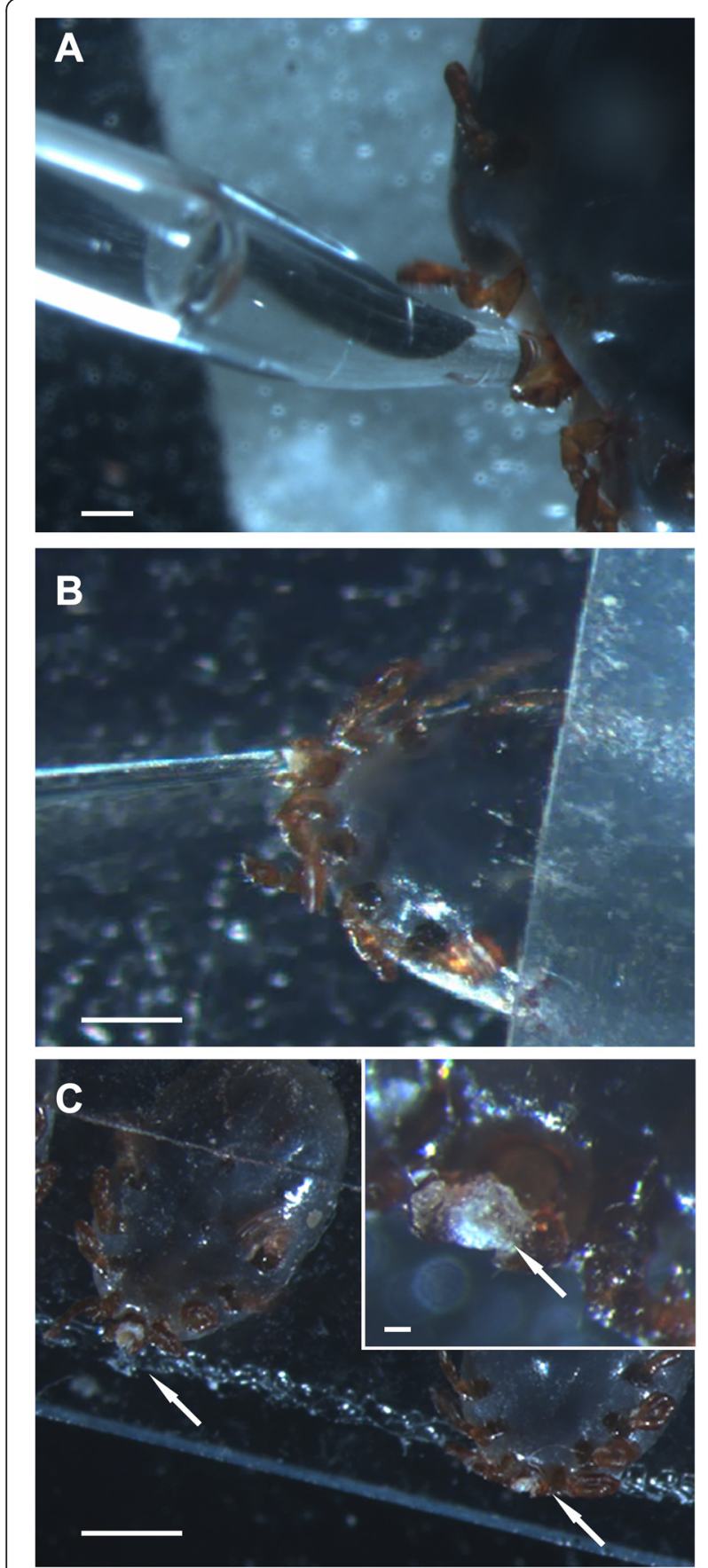

Fig. 1 Haemaphysalis longicornis saliva collection. a Saliva collection from a fully engorged adult female. b Saliva collection from a fully engorged nymph. c After application of dopamine, saliva appears as crystals in nymph mouthparts (arrows). Insert shows a magnified image of crystals in mouthparts. Bars $=100 \mu \mathrm{m}$

published tick sialomes [9]. In the set of tick specific proteins, 30 proteins were identified exclusively in nymph saliva, 74 proteins were identified exclusively in adult saliva, and 31 proteins were detected in both stages (Fig. 2). Of those 31 identified tick proteins detected in both stages,

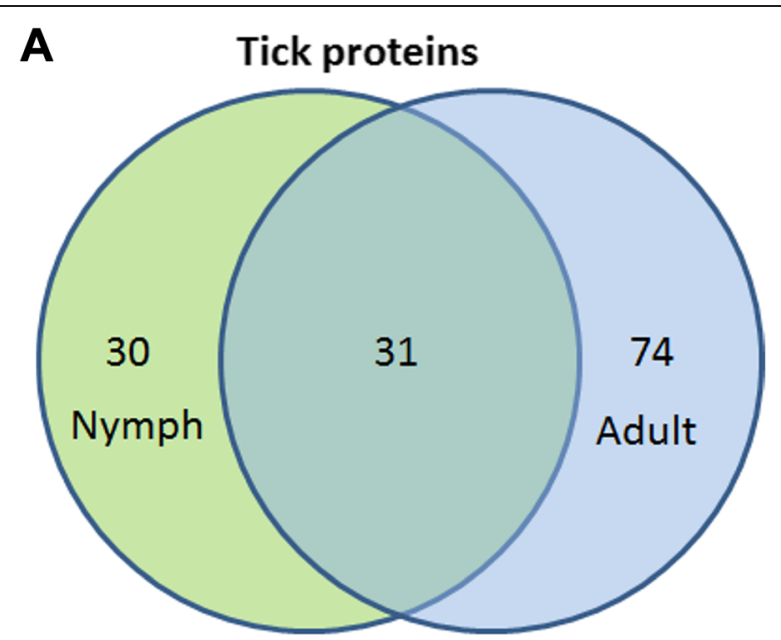

B Rabbit proteins

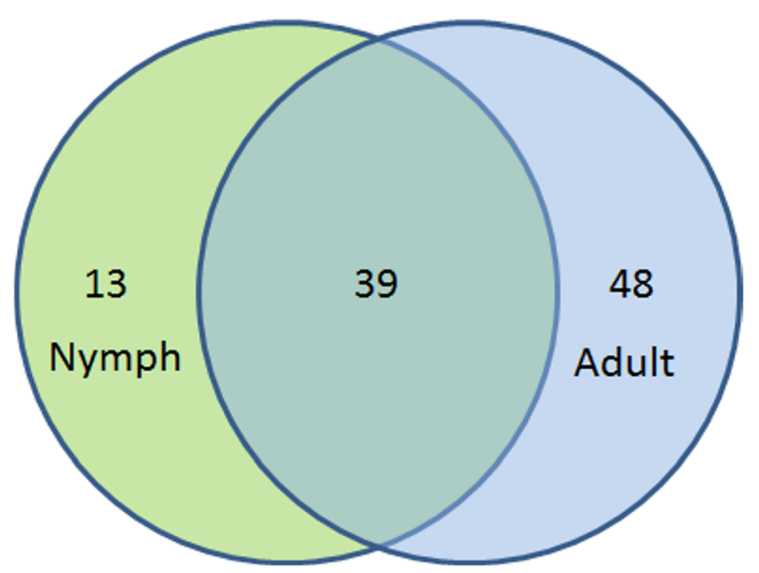

Fig. 2 Venn diagram of Haemaphysalis longicornis saliva proteins identified in fully engorged nymphs (nymph) and fully engorged adult females. a Tick and $\mathbf{b}$ rabbit proteins identified in tick saliva. The overlap between circles shows the proteins present in both stages

11 had statistical differential expression confirmed (Fig. 4 and Table 4). This finding is discussed below. These proteins identified in nymph saliva as well as in adult saliva can be secreted through developmental stages throughout the tick feeding process, since proteomic studies using saliva from other tick species collected during feeding process have described the presence of similar classes of proteins as identified in this study [14-16]. Moreover, some of these proteins have been used in anti-tick vaccination experiments, e.g. glutathione S-transferase [50, 51], cystatin [52], ferritin [53, 54], serpins [23, 55-59], and hemelipoproteins $[60,61]$.

The identified tick proteins include (i) heme/iron metabolism-related proteins, including hemelipoproteins and ferritin; (ii) proteins related to oxidation/detoxification functions, including glutathione S-transferase, aldehyde 
Table 1 Tick and host proteins identified exclusively in nymph saliva

\begin{tabular}{|c|c|c|c|}
\hline Contig number ${ }^{a}$ & Annotation & Class & Spec count \\
\hline \multicolumn{4}{|l|}{ TICK } \\
\hline BAF36722 & glycine-rich cell wall structural protein & Glycine-rich protein & 1.00 \\
\hline Rm-6837 & glycine proline-rich secreted protein & Glycine-rich protein & 5.25 \\
\hline Rm-21891 & actin-depolymerizing factor & Cytoskeletal & 3.00 \\
\hline JAA63693 & substrate adhesion-dependent cell spreading & Extracellular matrix/cell adhesion & 2.50 \\
\hline JAA55046 & toll-like receptor 5 & Immunity & 5.00 \\
\hline ACX33152 & catalytically inactive chitinase-like lectins & Metabolism, carbohydrate & 5.00 \\
\hline JAA63210 & lysosomal alpha-mannosidase-like isoform X2 & Metabolism, carbohydrate & 2.00 \\
\hline JAA54599 & phospholipase A2 & Metabolism, lipid & 6.00 \\
\hline Rm-32843 & lysosomal \& prostatic acid phosphatase & Protein modification machinery & 4.66 \\
\hline BAF31119 & secreted protein & Secreted conserved protein & 7.75 \\
\hline AEO33293 & antigen $5 / \mathrm{SCP}$ & Secreted conserved protein & 5.75 \\
\hline AEO36255 & secreted protein & Secreted conserved protein & 1.66 \\
\hline JAA60619 & secreted protein & Secreted conserved protein & 5.50 \\
\hline Rm-9069 & secreted protein & Secreted conserved protein & 1.50 \\
\hline BAE02551 & lipocalin & Lipocalin & 5.50 \\
\hline BAF43801 & longipain & Proteinase & 5.75 \\
\hline DAA34687 & cathepsin L-like & Proteinase & 1.50 \\
\hline JAA62284 & tick serine protease & Proteinase & 1.50 \\
\hline Rm-44814 & serine carboxypeptidase & Proteinase & 2.00 \\
\hline ACJ26770 & alpha-2-macroglobulin & Proteinase inhibitor & 27.75 \\
\hline EEC05896 & serpin & Proteinase inhibitor & 1.00 \\
\hline AEO34218 & serpin & Proteinase inhibitor & 2.33 \\
\hline AEO34349 & serpin & Proteinase inhibitor & 4.75 \\
\hline JAB70612 & alpha-2-macroglobulin & Proteinase inhibitor & 1.25 \\
\hline Rm-12491 & serpin & Proteinase inhibitor & 4.00 \\
\hline AEO35364 & cystatin & Proteinase inhibitor & 1.25 \\
\hline EEC02492 & TGF-beta-induced protein ig-h3 & Signal transduction & 5.25 \\
\hline JAA58175 & chorion peroxidase & Heme/iron metabolism & 5.50 \\
\hline BAN13552 & ferritin & Heme/iron metabolism & 5.00 \\
\hline JAA54618 & metabotropic glutamate receptor & Transporters/receptors & 2.25 \\
\hline \multicolumn{4}{|l|}{ RABBIT } \\
\hline G1T4V7 & myosin tail & Cytoskeletal & 6.25 \\
\hline G1T4A5 & collagen alpha-1 (I) chain-like & Extracellular matrix/cell adhesion & 4.00 \\
\hline P25227 & alpha-1-acid glycoprotein 1 & Immunity & 1.66 \\
\hline G1TJG6 & glyceraldehyde-3-phosphate dehydrogenase & Metabolism, energy & 1.50 \\
\hline G1TFX2 & alpha-1-antiproteinase & Proteinase inhibitor & 10.00 \\
\hline Q28665 & alpha-1-antiproteinase & Proteinase inhibitor & 10.00 \\
\hline G1SDN2 & keratin 17 & Keratin & 35.50 \\
\hline G1SHY2 & keratin 75 - extracellular vesicular exosome & Keratin & 71.00 \\
\hline G1SHZ4 & keratin type II cytoskeletal 7 & Keratin & 48.50 \\
\hline G1SPP3 & keratin type II cytoskeletal 4 & Keratin & 59.50 \\
\hline
\end{tabular}


Table 1 Tick and host proteins identified exclusively in nymph saliva (Continued)

\begin{tabular}{llll}
\hline G1SUH1 & keratin type II cytoskeletal 72 isoform X1 & Keratin & 7.66 \\
G1T8T1 & keratin type I cytoskeletal 28 & Keratin & 13.25 \\
G1U758 & keratin type I cytoskeletal 18 & Keratin & 16.25 \\
\hline
\end{tabular}

${ }^{a}$ Protein and spectral count of host and tick proteins identified in nymph Haemaphysalis longicornis saliva. Annotation and accession numbers of best match identities obtained using BLASTP against several protein databases can be checked with more details in Additional file 1: Table S1 and Additional file 2: Table S2

dehydrogenase, glutathione peroxidase, and catalase; (iii) enzymes such as serine proteinases, cysteine proteinases, metalloexopeptidases, carboxypeptidases, and aspartic proteases; (iv) proteinase inhibitors of the serpin, cystatin, alpha-2-macroglobulin, trypsin inhibitor-like (TIL), and Kunitz families; (v) tick-specific protein families including lipocalin (histamine-binding proteins), glycine-rich proteins, and the group of secreted conserved proteins [9]. In addition, proteins related to cytoskeletal functions, protein modification machinery, signal transduction, transporters and receptors, metabolism of carbohydrate, energy, nucleotide, amino acids, and lipid were identified (Fig. 3 and Tables 1, 2 and 3).

\section{Heme/iron metabolism-related proteins}

The most abundant proteins identified in $H$. longicornis saliva are hemelipoproteins, which are associated with heme/iron metabolic processes. This finding is in agreement with previous studies describing hemelipoproteins in proteomic saliva of Ornithodoros moubata, Rhipicephalus microplus, Ixodes scapularis [13, 16, 30, 31], as well as with a study about $A$. americanum immunoproteome [32], which showed that hemelipoproteins are the major proteins in tick saliva.

Hemelipoproteins were first described as heme-binding proteins from tick hemolymph, being able to transport cholesterol, phospholipids, and free fatty acids, in addition to heme $[62,63]$. These proteins are the most abundant proteins in spontaneously detached fully engorged $H$. longicornis saliva, which were relatively highly expressed in nymph saliva (Fig. 3 and Table 4). This data is in accordance with the findings observed for $R$. microplus, where hemelipoproteins were highly expressed in partially engorged adult ticks, showing a higher expression in the early developmental stages of tick feeding [16]. The physiological role of hemelipoproteins in blood meal acquisition is not completely understood. Tissue and vessel dilaceration produced by style of tick feeding and the presence of hemoglobin digestive enzymes (such as cathepsin and legumain) in tick saliva cause hemolysis and heme release in the feeding site. It is known that heme activates innate immune cells such as macrophages and neutrophils through activation of innate immune receptors [64-66], thus a role of hemelipoproteins being injected into the feeding site to prevent heme-induced inflammation is suggested. In addition, these proteins could be involved in a heme-excretory system, removing heme excess from tick and re-injecting it into the host. However, the presence of heme in tick saliva needs further investigation.

\section{Proteinases}

Several proteinases classes were identified in $H$. longicornis saliva: (i) serine proteinases; (ii) cysteine proteinases (including longipain, legumain and cathepsin L); (iii) aspartic proteinases; (iv) serine carboxypeptidases; and (v) proteins that belong to peptidase family M20 from MEROPS (including dipeptidases, and metalloexopeptidases). This set of different proteins present in tick saliva could have multiple modes of action during blood feeding. Serine proteinases may interfere with host inflammation and blood clotting. As shown by the presence of I. scapularis saliva protein $\mathrm{C}$ activator, a protein acting in the production of activated protein $\mathrm{C}$, a potent anticoagulant that also regulates a myriad of inflammatory responses through protease activated receptors activation [67].

Cysteine proteinases, aspartic proteinases, serine carboxypeptidases and legumains have been described mainly as digestive enzymes with a role in hemoglobin digestion $[68,69]$ and pathogen transmission in ticks [70]. Thus, the presence of these enzymes in the feeding site may indicate that they act as digestive enzymes secreted into the host, digesting blood components at the tick attachment site and facilitating pathogen transmission during tick feeding. The presence of putative digestive enzymes in tick saliva was also observed in $R$. microplus [16].

In general, dipeptidases hydrolyze the late products of protein degradation to complete the conversion of proteins to free amino acids. In ticks, dipeptidases were reported to be responsible for the destruction of bradykinin, a potent pain inductor [71]. These enzymes are related to the kininase activity found in I. scapularis saliva, which may be responsible for the lack of host pain response subsequent to attachment and feeding [72].

\section{Proteinase inhibitors}

Host defense responses triggered by tick feeding are mainly dependent on the action of several proteinases, such as procoagulant (thrombin, factor Xa and other coagulation factors), pro-inflammatory (neutrophil elastase, proteinase-3, chymase, tryptase, kallikrein, cathepsin L, cathepsin B, cathespin S, cathepsin C, and cathepsin G) and complement enzymes (factors B, C, D, and component 2) 
Table 2 Tick and host proteins identified exclusively in adult saliva

\begin{tabular}{|c|c|c|c|}
\hline Contig number $^{\mathrm{a}}$ & Annotation & Class & Spec count \\
\hline \multicolumn{4}{|l|}{ TICK } \\
\hline JAA54320 & glycine-rich protein & Glycine-rich protein & 1.50 \\
\hline EEC14126 & radixin/ezrin/moesin & Cytoskeletal & 2.00 \\
\hline ACX53929 & putative beta thymosin & Cytoskeletal & 1.33 \\
\hline DAA34555 & microtubule-binding protein & Cytoskeletal & 1.00 \\
\hline AEO32824 & actin depolymerizing factor & Cytoskeletal & 1.50 \\
\hline AEO33976 & beta tubulin & Cytoskeletal & 6.50 \\
\hline AFR32950 & paramyosin & Cytoskeletal & 5.33 \\
\hline JAB76162 & dynein light chain & Cytoskeletal & 1.00 \\
\hline JAB80373 & myosin class i heavy chain & Cytoskeletal & 12.25 \\
\hline AAY42205 & troponin $\mathrm{T}$ & Cytoskeletal & 1.00 \\
\hline Rm-1533 & beta tubulin partial & Cytoskeletal & 6.33 \\
\hline Rm-80704 & myosin class ii heavy chain & Cytoskeletal & 4.67 \\
\hline ACF35539 & glutathione S-transferase & Oxidant metabolism/detoxification & 6.50 \\
\hline ACG76272 & aldehyde dehydrogenase & Oxidant metabolism/detoxification & 1.00 \\
\hline AAQ74441 & glutathione S-transferase & Oxidant metabolism/detoxification & 1.50 \\
\hline AEO33057 & sulfotransferase & Oxidant metabolism/detoxification & 2.00 \\
\hline AEO35358 & catalase & Oxidant metabolism/detoxification & 8.00 \\
\hline JAA63098 & putative aldehyde dehydrogenase & Oxidant metabolism/detoxification & 3.00 \\
\hline Rm-14504 & aldehyde dehydrogenase & Oxidant metabolism/detoxification & 14.75 \\
\hline Rm-46289 & aldehyde dehydrogenase & Oxidant metabolism/detoxification & 2.00 \\
\hline ADN34303 & cuticular protein & Extracellular matrix/cell adhesion & 4.75 \\
\hline AEO34631 & phosphoserine aminotransferase & Metabolism, amino acid & 1.50 \\
\hline Rm-73466 & homocysteine S-methyltransferase & Metabolism, amino acid & 2.50 \\
\hline AEO32930 & transketolase & Metabolism, carbohydrate & 2.33 \\
\hline ACH88101 & glyceraldehyde-3-phosphate dehydrogenase & Metabolism, energy & 2.67 \\
\hline AEO32901 & ATP synthase subunit beta & Metabolism, energy & 7.75 \\
\hline AEO34579 & 3-phosphoglycerate dehydrogenase & Metabolism, energy & 3.75 \\
\hline AEO35473 & Isocitrate dehydrogenase NADP & Metabolism, energy & 6.00 \\
\hline JAA60091 & 3-phosphoglycerate dehydrogenase & Metabolism, energy & 3.33 \\
\hline JAA60302 & Isocitrate dehydrogenase NADP & Metabolism, energy & 7.00 \\
\hline JAA62224 & imp cyclohydrolase/methylglyoxal synthase & Metabolism, energy & 8.75 \\
\hline JAA66712 & C-1-tetrahydrofolate synthase & Metabolism, energy & 2.00 \\
\hline JAA68969 & glyceraldehyde-3-phosphate dehydrogenase & Metabolism, energy & 2.50 \\
\hline JAB74970 & cytochrome B5 & Metabolism, energy & 1.33 \\
\hline Rm-26196 & Malate dehydrogenase & Metabolism, energy & 2.67 \\
\hline $\mathrm{Rm}-18758$ & 15-hydroxyprostaglandin dehydrogenase & Metabolism, lipid & 1.50 \\
\hline Rm-73635 & farnesoic acid o-methyltransferase & Metabolism, lipid & 1.67 \\
\hline JAA59537 & $3^{\prime}\left(2^{\prime}\right) 5^{\prime}$-bisphosphate nucleotidase & Metabolism, nucleotide & 1.00 \\
\hline \multirow[t]{2}{*}{ JAA60240 } & GDP dissociation inhibitor & Metabolism, nucleotide & 1.50 \\
\hline & Inosine-5'-monophosphate dehydrogenase & Metabolism, nucleotide & 4.75 \\
\hline Rm-32846 & purine nucleoside phosphorylase transferase & Metabolism, nucleotide & 2.00 \\
\hline Rm-6587 & SAICAR synthase & Metabolism, nucleotide & 2.00 \\
\hline AEO35504 & annexin & Protein export machinery & 1.00 \\
\hline
\end{tabular}


Table 2 Tick and host proteins identified exclusively in adult saliva (Continued)

\begin{tabular}{|c|c|c|c|}
\hline BAF63673 & protein disulfide-isomerase & Protein modification machinery & 2.50 \\
\hline EEC14106 & multifunctional chaperone & Protein modification machinery & 2.50 \\
\hline JAA60036 & heat shock protein & Protein modification machinery & 1.50 \\
\hline JAA67522 & heat shock protein hsp 90-alpha isoform & Protein modification machinery & 2.50 \\
\hline JAB73342 & methyltransferase & Protein modification machinery & 1.50 \\
\hline JAB75807 & cysteine s-methyltransferase & Protein modification machinery & 2.00 \\
\hline Rm-24571 & heat shock protein 70 cognate & Protein modification machinery & 6.50 \\
\hline Rm-62470 & heat shock-related protein & Protein modification machinery & 3.75 \\
\hline JAA62377 & gmp synthase & Protein modification machinery & 2.50 \\
\hline AEO32591 & elongation factor 1 -alpha & Protein synthesis machinery & 8.33 \\
\hline AEO32633 & AAA+ ATPase & Protein synthesis machinery & 1.00 \\
\hline Rm-50382 & elongation factor 2-like & Protein synthesis machinery & 4.00 \\
\hline AEO34773 & secreted protein & Secreted conserved protein & 3.00 \\
\hline AEO32884 & lipocalin & Lipocalin & 7.25 \\
\hline Rm-26539 & lipocalin & Lipocalin & 5.00 \\
\hline BAF51711 & tick legumain & Proteinase & 8.50 \\
\hline BAH86062 & cathepsin L-like & Proteinase & 2.33 \\
\hline JAA60171 & metalloexopeptidase & Proteinase & 3.00 \\
\hline JAA65860 & CNDP dipeptidase & Proteinase & 2.33 \\
\hline EEC20000 & alpha-2-macroglobulin & Proteinase inhibitor & 6.00 \\
\hline BAH02683 & haemalin & Proteinase inhibitor & 4.50 \\
\hline AEO35673 & TIL & Proteinase inhibitor & 5.00 \\
\hline BAG41813 & cyclophilin 1 & Signal transduction & 3.50 \\
\hline AEO32842 & regulatory protein $\mathrm{mlp}$ & Signal transduction & 2.00 \\
\hline JAB75372 & calmodulin & Signal transduction & 1.67 \\
\hline Rm-69447 & neuropeptide-like protein 31 & Signal transduction & 1.00 \\
\hline Rm-79606 & Regucalcin & Signal transduction & 2.50 \\
\hline Rm-154439 & hemelipoprotein & Heme/iron metabolism & 1.67 \\
\hline JAA62029 & zinc finger protein & Transcription machinery & 1.00 \\
\hline EEC05877 & apolipophorin & Transporters/receptors & 2.75 \\
\hline JAB78095 & glutamate receptor 1-like & Transporters/receptors & 1.67 \\
\hline \multicolumn{4}{|l|}{ RABBIT } \\
\hline G1U9R8 & gelsolin & Cytoskeletal & 2.50 \\
\hline P27170 & serum paraoxonase/arylesterase 1 & Oxidant metabolism/detoxification & 1.00 \\
\hline G1SR65 & myeloperoxidase & Oxidant metabolism/detoxification & 1.50 \\
\hline G1T049 & collagen alpha-6(IV) chain & Extracellular matrix/cell adhesion & 1.00 \\
\hline G1TZA1 & type $X$ collagen alpha 1-like & Extracellular matrix/cell adhesion & 2.25 \\
\hline O77791 & protein S100-A12 & Immunity & 4.50 \\
\hline P01840 & Ig kappa-b4 chain C region & Immunity & 10.25 \\
\hline P01870 & Ig gamma-1 chain $C$ region & Immunity & 48.50 \\
\hline P04221 & Ig mu chain $\mathrm{C}$ region & Immunity & 4.00 \\
\hline G1SS91 & complement C4-A & Immunity & 3.75 \\
\hline G1T3X1 & complement component C9 & Immunity & 2.00 \\
\hline G1TEC1 & Ig kappa chain V-I & Immunity & 4.75 \\
\hline G1TFU1 & Ig kappa chain V-I & Immunity & 5.25 \\
\hline
\end{tabular}


Table 2 Tick and host proteins identified exclusively in adult saliva (Continued)

\begin{tabular}{|c|c|c|c|}
\hline G1TKP3 & immunoglobulin lambda-like & Immunity & 4.00 \\
\hline G1TM51 & Ig kappa chain V-I & Immunity & 4.00 \\
\hline G1TPF2 & T-cell surface glycoprotein CD8 -like & Immunity & 5.00 \\
\hline G1TPZ1 & galectin-1 & Immunity & 2.33 \\
\hline G1TRW8 & neutrophil gelatinase-associated lipocalin & Immunity & 8.50 \\
\hline G1TUX5 & C1q and TNF related protein 7-like & Immunity & 2.50 \\
\hline G1TVN7 & Ig kappa chain V-I & Immunity & 5.75 \\
\hline G1TVZ5 & T-cell surface glycoprotein CD8 -like & Immunity & 1.50 \\
\hline Q9GK63 & mammaglobin-B & Immunity & 2.00 \\
\hline Q9GK67 & secretoglobin & Immunity & 3.67 \\
\hline U3KM01 & Ig kappa chain V-I & Immunity & 4.00 \\
\hline G1SQA8 & ATP synthase mitochondrial precursor & Metabolism, energy & 3.50 \\
\hline G1TS29 & triosephosphate isomerase & Metabolism, energy & 1.33 \\
\hline G1TYA7 & L-lactate dehydrogenase $B$ chain & Metabolism, energy & 2.67 \\
\hline G1TB24 & peroxysomal Fatty Acyl CoA Transporter & Metabolism, lipid & 1.50 \\
\hline G1SN21 & purine nucleoside phosphorylase & Metabolism, nucleotide & 2.00 \\
\hline G1TXI1 & nucleophosmin & Metabolism, nucleotide & 3.33 \\
\hline G1U9T4 & nucleoside diphosphate kinase B & Metabolism, nucleotide & 3.33 \\
\hline G1U155 & histone $\mathrm{H} 2 \mathrm{~B}$ type 1 -like & Nuclear regulation & 3.00 \\
\hline P51662 & annexin A1 & Protein export machinery & 5.50 \\
\hline G1TDI4 & transthyretin & Protein export machinery & 3.00 \\
\hline G1SJZ9 & thioredoxin & Protein modification machinery & 1.33 \\
\hline G1SEK8 & metalloendopeptidase inhibitor & Proteinase inhibitor & 6.75 \\
\hline G1SIKO & antithrombin III & Proteinase inhibitor & 8.67 \\
\hline G1SZA4 & inter-alpha-trypsin inhibitor heavy chain $\mathrm{H} 1$ & Proteinase inhibitor & 2.00 \\
\hline G1TM88 & alpha-1-antitrypsin & Proteinase inhibitor & 2.33 \\
\hline G1U6R8 & negative regulation of endopeptidase & Proteinase inhibitor & 8.25 \\
\hline Q45GR2 & alpha-2-antiplasmin & Proteinase inhibitor & 3.50 \\
\hline G1SNP6 & serine/threonine-protein kinase & Signal transduction & 1.50 \\
\hline P09809 & apolipoprotein A-I & Heme/iron metabolism & 4.25 \\
\hline G1TFW8 & lactotransferrin & Heme/iron metabolism & 5.75 \\
\hline G1SU82 & vitamin D-binding protein & Transporters/receptors & 4.75 \\
\hline G1T4K1 & sodium channel protein type 3 isoform $X_{1}$ & Transporters/receptors & 2.00 \\
\hline G1TP66 & keratin type II cuticular $\mathrm{Hb} 3$ & Keratin & 1.50 \\
\hline P00919 & carbonic anhydrase 2 & Hemoglobin/RBC products & 3.50 \\
\hline
\end{tabular}

aProtein and spectral count of host and tick proteins identified in adult Haemaphysalis longicornis saliva. Annotation and accession numbers of best match identities obtained using BLASTP against the several protein databases can be checked with more details in Additional file 1: Table S1 and Additional file 2: Table S2

[9-12]. Proteinases from these pathways are regulated by specific endogenous inhibitors, maintaining homeostasis. From this perspective, proteinase inhibitors secreted into the feeding site act by disrupting host defenses, facilitating blood meal acquisition. Several families of protease inhibitor domains were found in saliva of $H$. longicornis (Tables 1, 2 and 3).

Serpins are a superfamily of serine proteinase inhibitors involved in blood coagulation, fibrinolysis, inflammation, and complement activation in mammals [73, 74]. These proteins were found mostly in nymph saliva (Table 1). Tick serpins are secreted into the feeding site to disrupt host defenses against tick feeding, including anticoagulant [75-77] and immunomodulatory responses [78-80]. The potential effect of these proteins on host systems is supported by several studies that demonstrate the mortality and reduced feeding efficiency when several tick species were fed on host immunized with tick recombinant 
Table 3 Tick and host proteins identified in nymph and adult saliva

\begin{tabular}{|c|c|c|c|c|}
\hline \multirow[t]{2}{*}{ Contig number } & \multirow[t]{2}{*}{ Annotation } & \multirow[t]{2}{*}{ Class } & \multicolumn{2}{|c|}{ Spec count } \\
\hline & & & Nymph & Adult \\
\hline \multicolumn{5}{|l|}{ TICK } \\
\hline ABQ96858 & tropomyosin & Cytoskeletal & 3.00 & 5.25 \\
\hline BAF98180 & actin & Cytoskeletal & 21.75 & 18.75 \\
\hline AEO32669 & alpha tubulin & Cytoskeletal & 3.25 & 11.00 \\
\hline AGC13075 & glutathione peroxidase & Oxidant metabolism/detoxification & 10.50 & 4.33 \\
\hline AEO34612 & enolase & Metabolism, carbohydrate & 2.00 & 4.25 \\
\hline Rm-10851 & ATP synthase subunit alpha & Metabolism, energy & 1.50 & 4.67 \\
\hline AEO34838 & histone $\mathrm{H} 4$ & Nuclear regulation & 5.75 & 7.33 \\
\hline AEO34879 & histone $\mathrm{H} 2 \mathrm{~A}$ & Nuclear regulation & 4.00 & 10.00 \\
\hline AEO32095 & heat shock $70 \mathrm{kDa}$ protein & Protein modification machinery & 5.50 & 5.67 \\
\hline AEO32791 & heat shock protein & Protein modification machinery & 9.75 & 11.00 \\
\hline AEO34048 & protein disulfide-isomerase A6-like & Protein modification machinery & 1.50 & 1.00 \\
\hline JAA62581 & heat shock protein & Protein modification machinery & 3.00 & 3.50 \\
\hline ADG86641 & lysosomal acid phosphatase & Protein modification machinery & 9.50 & 26.75 \\
\hline JAA73257 & ubiquitin/40s ribosomal protein s27a & Proteasome machinery & 17.25 & 4.75 \\
\hline ADK47399 & secreted protein & Secreted conserved protein & 2.67 & 3.00 \\
\hline AGH08176 & AV422 & Secreted conserved protein & 4.75 & 4.25 \\
\hline BAE53722 & aspartic protease & Proteinase & 4.00 & 3.25 \\
\hline BAD11156| & serpin & Proteinase inhibitor & 15.50 & 3.50 \\
\hline JAA60430 & alpha-2-macroglobulin & Proteinase inhibitor & 41.00 & 12.33 \\
\hline JAA64973 & alpha-2-acroglobulin & Proteinase inhibitor & 17.50 & 3.50 \\
\hline Rm-7619 & alpha-2-macroglobulin & Proteinase inhibitor & 26.25 & 9.00 \\
\hline ABZ89554. & cystatin & Proteinase inhibitor & 6.33 & 2.67 \\
\hline Rm-69112 & 14-3-3 zeta & Signal transduction & 4.33 & 2.33 \\
\hline BAG12081 & hemelipoprotein & Heme/iron metabolism & 796.25 & 323.25 \\
\hline BAJ21514 & hemelipoprotein & Heme/iron metabolism & 405.00 & 224.00 \\
\hline BAJ21515 & hemelipoprotein & Heme/iron metabolism & 98.50 & 6.25 \\
\hline BAL42280 & hemelipoprotein & Heme/iron metabolism & 141.75 & 60.75 \\
\hline JAA59652 & hemelipoprotein & Heme/iron metabolism & 19.67 & 14.25 \\
\hline ABD83654 & hemelipoprotein & Heme/iron metabolism & 12.50 & 12.25 \\
\hline Rm-72548 & hemelipoprotein & Heme/iron metabolism & 32.25 & 14.75 \\
\hline JAA61676 & plexins functional semaphorin receptor & Transporters/receptors & 12.50 & 2.50 \\
\hline \multicolumn{5}{|l|}{ RABBIT } \\
\hline G1T229 & filaggrin-2 & Cytoskeletal & 17.00 & 1.50 \\
\hline G1T6W7 & catalase & Oxidant metabolism/detoxification & 5.25 & 7.50 \\
\hline P16973 & lysozyme C & Immunity & 3.00 & 7.50 \\
\hline P25230 & antimicrobial protein CAP18 & Immunity & 1.50 & 5.33 \\
\hline P50117 & protein S100-A9 & Immunity & 3.75 & 3.00 \\
\hline G1SUZ1 & complement C3 & Immunity & 5.25 & 15.00 \\
\hline G1SYM4 & alpha-1B-glycoprotein & Immunity & 3.75 & 3.00 \\
\hline G1THZ6 & Ig gamma-1 chain $C$ region & Immunity & 15.00 & 62.00 \\
\hline G1T0Z2 & histone $\mathrm{H} 2 \mathrm{~A}$ type 1-A & Nuclear regulation & 4.00 & 5.67 \\
\hline G1T9M9 & heat shock cognate $71 \mathrm{kDa}$ protein & Protein modification machinery & 9.00 & 10.00 \\
\hline
\end{tabular}


Table 3 Tick and host proteins identified in nymph and adult saliva (Continued)

\begin{tabular}{|c|c|c|c|c|}
\hline G1SQ70 & alpha-2-macroglobulin & Proteinase inhibitor & 10.75 & 32.50 \\
\hline G1TFV7 & alpha-1-antiproteinase & Proteinase inhibitor & 10.25 & 37.25 \\
\hline Q07298 & alpha-1-antiproteinase & Proteinase inhibitor & 10.00 & 32.67 \\
\hline P19134 & serotransferrin & Heme/iron metabolism & 33.75 & 95.25 \\
\hline P20058 & hemopexin & Heme/iron metabolism & 12.00 & 36.50 \\
\hline G1SQ02 & peroxiredoxin-1 & Heme/iron metabolism & 3.00 & 3.25 \\
\hline G1SWF6 & haptoglobin - hemoglobin binding & Heme/iron metabolism & 7.50 & 20.00 \\
\hline G1TVS4 & hemopexin & Heme/iron metabolism & 12.00 & 38.25 \\
\hline G1U9S2 & serum albumin & Heme/iron metabolism & 156.00 & 665.50 \\
\hline G1SGQ5 & alpha-2-HS-glycoprotein & Transporters/receptors & 3.00 & 1.50 \\
\hline G1SLY0 & HCO3- transporter family & Transporters/receptors & 1.50 & 3.00 \\
\hline U3KMC6 & ceruloplasmin & Transporters/receptors & 3.67 & 8.50 \\
\hline G1SKE3 & keratin type II cytoskeletal 6A & Keratin & 84.50 & 20.00 \\
\hline G1SUH8 & keratin 2 & Keratin & 228.75 & 52.00 \\
\hline G1SWB8 & keratin type I cytoskeletal 27 & Keratin & 23.67 & 14.00 \\
\hline G1SY72 & keratin type II cytoskeletal & Keratin & 68.50 & 17.33 \\
\hline G1T1V0 & keratin type I cytoskeletal 10 & Keratin & 108.50 & 58.50 \\
\hline G1T1Y7 & keratin type I cytoskeletal 14 isoform X1 & Keratin & 56.00 & 12.50 \\
\hline G1T4R6 & keratin type I cytoskeletal 16 & Keratin & 57.50 & 13.25 \\
\hline G1T4S1 & keratin 15 & Keratin & 51.75 & 17.00 \\
\hline G1TDN6 & keratin type II cytoskeletal 5 & Keratin & 99.75 & 14.75 \\
\hline G1U754 & histidine-rich glycoprotein & Heme/iron metabolism & 11.75 & 25.25 \\
\hline G1U918 & keratin type II cytoskeletal 1 & Keratin & 104.25 & 39.75 \\
\hline G1T0W8 & fibrinogen beta chain & Fibrinogen & 5.25 & 26.50 \\
\hline G1T0X2 & fibrinogen alpha chain & Fibrinogen & 9.00 & 13.50 \\
\hline G1TKX3 & fibrinogen gamma chain & Fibrinogen & 6.25 & 34.75 \\
\hline P01948 & hemoglobin subunit alpha-1/2 & Hemoglobin/RBC products & 87.00 & 544.00 \\
\hline P02057 & hemoglobin subunit beta & Hemoglobin/RBC products & 100.75 & 517.50 \\
\hline P07452 & carbonic anhydrase 1 & Hemoglobin/RBC products & 5.00 & 11.00 \\
\hline
\end{tabular}

aprotein and spectral count of host and tick proteins identified both in nymph and adult Haemaphysalis longicornis saliva. Annotation and accession numbers of best match identities obtained using BLASTP against several protein databases can be checked with more details in Additional file 1: Table S1 and Additional file 2: Table S2

serpins [55-59]. Additionally, serpins in Aedes aegypti and Anopheles stephensi have been shown to play a role in pathogen transmission $[81,82]$.

Cystatins form a large superfamily of reversible and tight-binding inhibitors that interact with papain-like cysteine proteases and legumains [83]. Tick salivary cystatins have been described as immunosuppressive and anti-inflammatory proteins [84-87]. Moreover, the importance of cystatins in tick physiology was observed in studies that showed that neutralization of cystatin reduces tick feeding ability [52, 85, 88, 89].

Haemalin is a member of Kunitz-type inhibitors identified in $H$. longicornis saliva. This protein has been described as a thrombin inhibitor, delaying bovine plasma clotting time and inhibiting both thrombin-induced fibrinogen clotting and thrombin-induced platelet aggregation. This protein was described as a midgut protein [29], and this is the first time it is described in H. longicornis saliva. Taking into account haemalin function, we suggested that this protein acts as an anticoagulant salivary protein during tick feeding.

TIL (trypsin inhibitor-like) proteins have been reported in several tick sialomes $[9,10]$ and are described as elastase inhibitors, which also have antimicrobial activity [90, 91]. Alpha-2-macroglobulin are a group of proteins that have been found to inhibit several serum proteinases in vertebrates, including thrombin, factor Xa and kallikreins [77, 92-94], mediating T-cell proliferation and activating macrophages [95, 96]. Thus, as these proteins are secreted both in nymph and in adult tick 


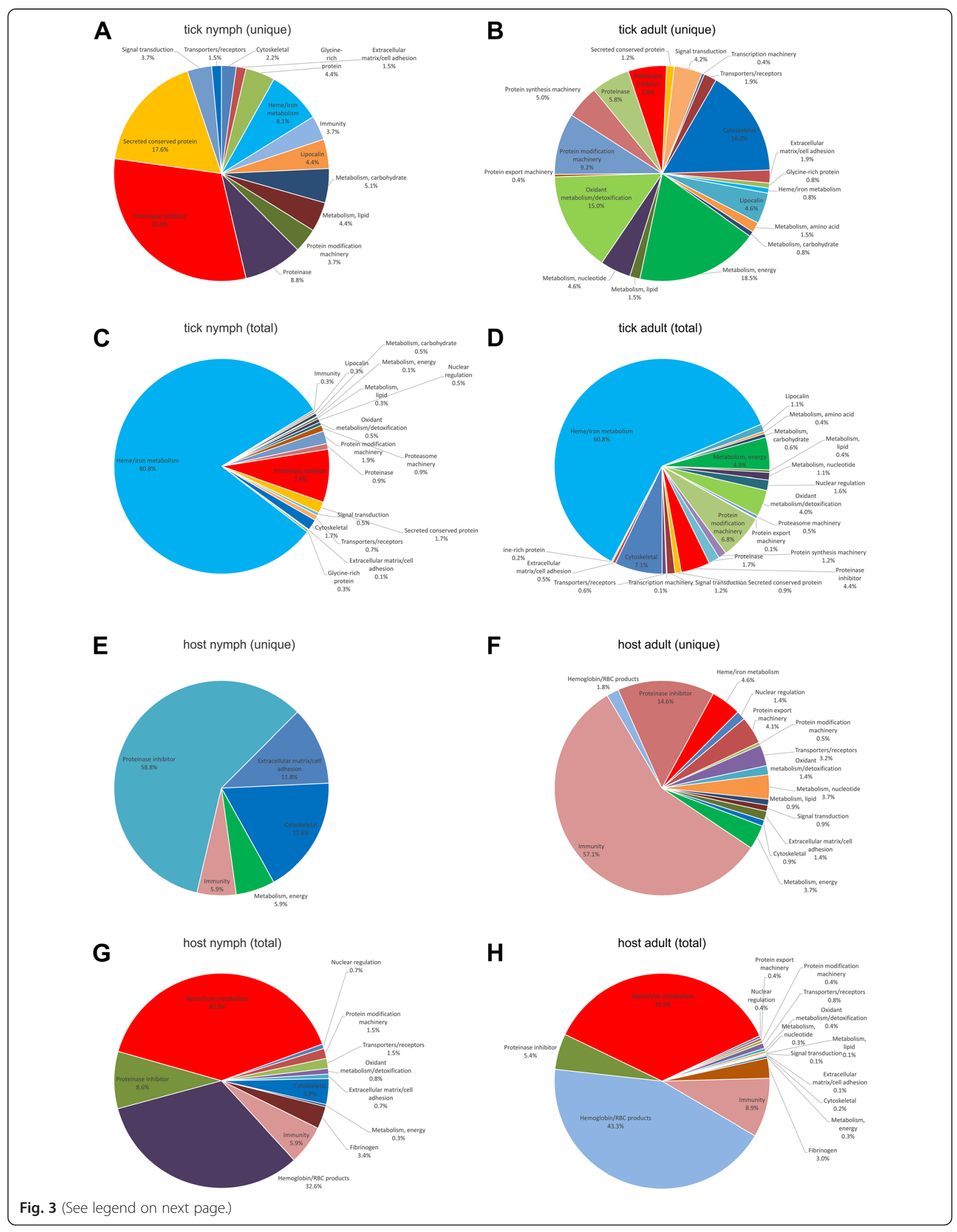


(See figure on previous page.)

Fig. 3 Functional classification of Haemaphysalis longicornis salivary proteins identified in fully engorged nymph (nymph) and fully engorged adult females (adult). a Tick proteins detected in nymph ( $\mathbf{a}$ and $\mathbf{c}$ ) and adult (b and $\mathbf{d}$ ). Proteins were divided as detected both in nymph (c) and adult (d) and proteins detected exclusively in nymph (a) or adult (b) and classified in groups according to their function and/or protein family. Pie charts represent the percentage of proteins found in each group with respect to normalized spectral counting for each sample. $\mathbf{b}$ Host proteins detected in nymph (e and $\mathbf{g}$ ) and adult ( $\mathbf{f}$ and $\mathbf{h}$ ). Proteins were divided as detected both in nymph $(\mathbf{g})$ and adult (h) and proteins detected exclusively in nymph (e) or adult (f) and classified in groups according to their function and/or protein family. Pie charts represent the percentage of proteins found in each group with respect to normalized spectral counting for each sample. Keratin was removed before data interpretation. (Additional file 3: Figure S1 shows data with keratin)

saliva, they could act as anticoagulant and/or immunomodulatory proteins during blood feeding.

\section{Tick-specific protein families}

Advances in transcriptomic and proteomic studies of tick salivary gland have created new opportunities to identify the variety of tick salivary transcripts and proteins. Many proteins are described to have no similarities to non-tick proteins from the NCBI database [9]. The secreted conserved protein group is composed mainly of tick proteins containing a signal peptide predicted and with similarities to proteins identified in other ticks [9]. Most of proteins included in this group have unknown functions and were described only in gene or protein sequencing projects, having their expression up-regulated after blood acquisition [97, 98]. A functionally described member of tick secreted conserved protein group is $A$. americanum AV422 protein. This protein was first described as an up-regulated protein in response to tick

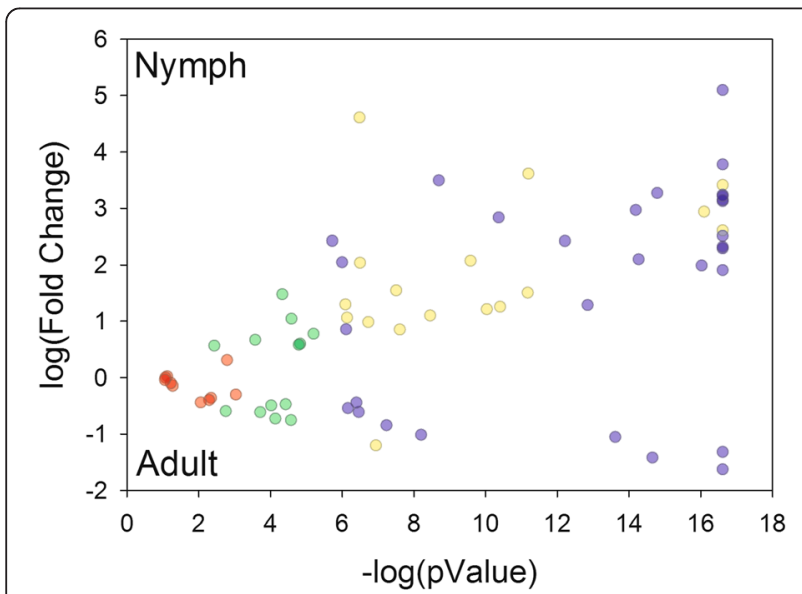

Fig. 4 Volcano plot abundance changes analysis of Haemaphysalis longicornis saliva proteins identified both in fully engorged nymph (nymph) and fully engorged adult females (adult). Each point represents the difference in expression (log2 fold difference) between nymph and adult plotted against the level of statistical significance. Proteins represented by (blue dot) had an identification that satisfied both fold and statistical criteria; (yellow dot) had an identifications that was filtered out by the L-stringency; (green dot) had an identification satisfied the fold criteria but, most likely, this happened by chance; and (red dot) had identification did not meet the fold and $p$-value criteria host exposure and/or to feeding stimuli in rabbits [99]. AV422 is secreted into the host during tick feeding, acting as an anticoagulant and anti-complement protein $[100,101]$. In $H$. longicornis, this protein is identified in both the spontaneously detached fully engorged nymph and adult saliva (Table 3). Additionally, an AV422-like protein was identified in partially and fully engorged $R$. microplus female saliva proteome [16]. Based on the high amino acid conservation and on its presence in other tick proteomes, it will be interesting to determine whether or not $H$. longicornis AV422-like protein is functionally similar to that of $A$. americanum.

The antigen 5 protein family is a group of cysteinerich secreted proteins [102]. This group is described in the salivary glands of blood-sucking insects and ticks, with functions that remain mostly unknown $[10,103,104]$, being identified exclusively in nymph saliva (Table 1). Glycine-rich proteins are extracellular matrix proteins and/or structural proteins with an important role in attachment to the host, since it they are present in cement material secreted by salivary glands during feeding process $[105,106]$. The expression levels of these proteins are associated with size of mouthparts and the number of hosts used by tick during its life cycle. Ticks with short mouthparts and one-host ticks express more glycine-rich protein. It could be implied that one-host ticks are more consistently pressed to sustain attachment on the host's skin [107]. In this sense, glycine-rich proteins were identified mostly in nymph saliva (Table 1). These proteins are also involved in the defense against pathogens, since they are inducible antibacterial proteins predominantly active by Gram-negative bacteria presents in insect and tick hemolymph and salivary gland [108].

Tick proteins of the lipocalin family are classified based on their homology with tick proteins containing the characteristic tick histamine-binding domain (PF02098) $[109,110]$. They are a group of multifunctional secreted proteins that bind several types of small hydrophobic ligands. The role as transport proteins is well studied, however it is clear that the arthropod lipocalin family is involved in various other physiological functions such as cell growth and metabolism, regulation of the immune response, and tissue repair [111]. Lipocalins were detected in several tick saliva proteomes $[13,16]$, and $H$. longicornis 
Table 4 Differential abundance of nymph and adult saliva proteins (determined using the TFold)

\begin{tabular}{|c|c|c|c|c|}
\hline Annotation & Contig number $^{a}$ & Class & Fold change ${ }^{b}$ & $p$ value \\
\hline \multicolumn{5}{|l|}{ TICK } \\
\hline Actin & BAF98180 & Cytoskeletal & 2.485 & 0.0001 \\
\hline glutathione peroxidase & AGC13075 & Oxidant metabolism/detoxification & 5.464 & 0.0002 \\
\hline histone $\mathrm{H} 4$ & AEO34838 & Nuclear regulation & 1.845 & 0.0145 \\
\hline ubiquitin/40s ribosomal protein s27a & JAA73257.1 & Proteasome machinery & 7.980 & 0.0000 \\
\hline serpin & BAD11156 & Proteinase inhibitor & 8.991 & 0.0000 \\
\hline cystatin & ABZ89554 & Proteinase inhibitor & 5.470 & 0.0190 \\
\hline hemelipoprotein & BAG12081 & Heme/iron metabolism & 5.089 & 0.0000 \\
\hline hemelipoprotein & BAJ21514 & Heme/iron metabolism & 3.823 & 0.0000 \\
\hline hemelipoprotein & BAJ21515 & Heme/iron metabolism & 34.697 & 0.0000 \\
\hline hemelipoprotein & BAL42280 & Heme/iron metabolism & 4.997 & 0.0000 \\
\hline hemelipoprotein & $\mathrm{Rm}-72548$ & Heme/iron metabolism & 4.371 & 0.0000 \\
\hline
\end{tabular}

RABBIT

\begin{tabular}{|c|c|c|c|c|}
\hline Ig gamma- 1 chain $C$ region & G1THZ6 & Immunity & -1.975 & 0.0034 \\
\hline alpha-1-antiproteinase & G1TFV7 & Proteinase inhibitor & -1.757 & 0.0066 \\
\hline serotransferrin & P19134 & Heme/iron metabolism & -1.328 & 0.0119 \\
\hline hemopexin & P20058 & Heme/iron metabolism & -1.423 & 0.0140 \\
\hline hemopexin & G1TVS4 & Heme/iron metabolism & -1.493 & 0.0113 \\
\hline serum albumin & G1U9S2 & Heme/iron metabolism & -2.028 & 0.0000 \\
\hline keratin type II cytoskeletal 6A & G1SKE3 & Keratin & 8.889 & 0.0000 \\
\hline keratin 2 & G1SUH8 & Keratin & 9.480 & 0.0000 \\
\hline keratin type I cytoskeletal 27 & G1SWB8 & Keratin & 4.210 & 0.0157 \\
\hline keratin type II cytoskeletal & G1SY72 & Keratin & 9.613 & 0.0000 \\
\hline keratin type I cytoskeletal 10 - & G1T1V0 & Keratin & 4.046 & 0.0000 \\
\hline keratin type I cytoskeletal 14 & G1T1Y7 & Keratin & 11.478 & 0.0024 \\
\hline keratin type I cytoskeletal 16 & G1T4R6 & Keratin & 9.827 & 0.0000 \\
\hline keratin 15 & G1T4S1 & Keratin & 7.271 & 0.0007 \\
\hline keratin type II cytoskeletal 5 & G1TDN6 & Keratin & 13.976 & 0.0000 \\
\hline keratin type II cytoskeletal 1 & G1U9|8 & Keratin & 5.796 & 0.0000 \\
\hline fibrinogen gamma chain & G1TKX3 & Fibrinogen & -2.612 & 0.0000 \\
\hline hemoglobin subunit alpha-1/2 & P01948 & Hemoglobin/RBC products & -3.021 & 0.0000 \\
\hline hemoglobin subunit beta & P02057 & Hemoglobin/RBC products & -2.440 & 0.0000 \\
\hline
\end{tabular}

TICK

heat shock protein

secreted protein

AV422

aspartic protease

alpha-2-macroglobulin

alpha-2-acroglobulin

alpha-2-macroglobulin

14-3-3 zeta

hemelipoprotein

hemelipoprotein

plexins functional semaphorin

AEO32791
ADK47399
AGH08176
BAE53722
JAA60430
JAA64973
Rm-7619
Rm-69112
JAA59652
ABD83654
JAA61676

Protein modification machinery

$1.839 \quad 0.0051$

Secreted conserved protein

$2.127 \quad 0.0143$

Secreted conserved protein

$2.434 \quad 0.0007$

Proteinase

$2.506 \quad 0.0147$

Proteinase inhibitor

Proteinase inhibitor

Proteinase inhibitor

7.808

12.469

0.0000

Signal transduction

Heme/iron metabolism

6.210

4.292

2.899

0.0004

Heme/iron metabolism

2.186

10.845

Transporters/receptors

0.0013

0.0004

0.0028

0.0000 
Table 4 Differential abundance of nymph and adult saliva proteins (determined using the TFold) (Continued)

\begin{tabular}{|c|c|c|c|c|}
\hline \multicolumn{5}{|l|}{ RABBIT } \\
\hline filaggrin-2 & G1T229 & Cytoskeletal & 24.880 & 0.0112 \\
\hline protein S100-A9 & P50117 & Immunity & 2.361 & 0.0009 \\
\hline alpha-1B-glycoprotein & G1SYM4 & Immunity & 2.983 & 0.0055 \\
\hline peroxiredoxin-1 & G1SQ02 & Heme/iron metabolism & 2.017 & 0.0094 \\
\hline alpha-2-HS-glycoprotein & G1SGQ5 & Transporters/receptors & 4.184 & 0.0111 \\
\hline fibrinogen beta chain & G1T0W8 & Fibrinogen & -2.248 & 0.0081 \\
\hline \multicolumn{5}{|l|}{ TICK } \\
\hline heat shock $70 \mathrm{kDa}$ protein & AEO32095 & Protein modification machinery & 2.101 & 0.0418 \\
\hline alpha tubulin & AEO32669 & Cytoskeletal & -1.649 & 0.0422 \\
\hline protein disulfide-isomerase & AEO34048 & Protein modification machinery & 2.844 & 0.0498 \\
\hline heat shock protein & JAA62581 & Protein modification machinery & 1.620 & 0.0842 \\
\hline ATP synthase subunit alpha & $\mathrm{Rm}-10851$ & Metabolism energy & -1.475 & 0.1486 \\
\hline \multicolumn{5}{|l|}{ RABBIT } \\
\hline antimicrobial protein CAP18 & P25230 & Immunity & -1.493 & 0.0764 \\
\hline alpha-2-macroglobulin & G1SQ70 & Proteinase inhibitor & -1.357 & 0.0467 \\
\hline complement C3 & G1SUZ1 & Immunity & -1.377 & 0.0617 \\
\hline fibrinogen alpha chain & G1T0X2 & Fibrinogen & 1.546 & 0.0353 \\
\hline histone $\mathrm{H} 2 \mathrm{~A}$ type $1-\mathrm{A}$ & G1T0Z2 & Nuclear regulation & 1.509 & 0.1858 \\
\hline catalase & G1T6W7 & Oxidant metabolism/detoxification & 1.525 & 0.0362 \\
\hline heat shock cognate $71 \mathrm{kDa}$ & G1T9M9 & Protein modification machinery & 1.747 & 0.0272 \\
\hline alpha-1-antiproteinase & Q07298 & Proteinase inhibitor & -1.618 & 0.0571 \\
\hline
\end{tabular}

TICK

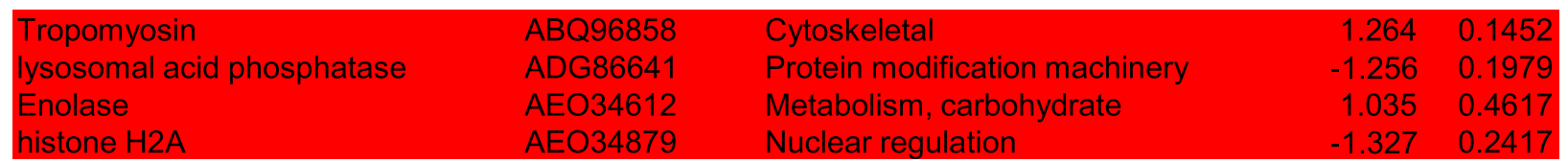

RABBIT

\begin{tabular}{|c|c|c|c|c|}
\hline carbonic anhydrase 1 & P07452 & Hemoglobin/RBC products & -1.048 & 0.4302 \\
\hline lysozyme C & P16973 & Immunity & -1.287 & 0.2053 \\
\hline HCO3- transporter family & G1SLY0 & Transporters/receptors & 1.020 & 0.4774 \\
\hline haptoglobin - hemoglobin binding & G1SWF6 & Heme/iron metabolism & -1.205 & 0.1225 \\
\hline histidine-rich glycoprotein & G1U754 & Heme/iron metabolism & -1.006 & 0.4825 \\
\hline Ceruloplasmin & U3KMC6 & Transporters/receptors & -1.081 & 0.4154 \\
\hline
\end{tabular}

Blue: identifications that satisfied both fold and statistical criteria

Yellow: identifications were filtered out by the L-stringency

Green: identifications satisfied the fold criteria but, most likely, this happened by chance

Red: identifications did not meet the fold and $p$-value criteria

${ }^{a}$ Accession numbers of best matches identities obtained using BLASTP against the non-redundant protein database in GenBank

${ }^{b}$ Positive number means the fold increased expression in nymph (relation between nymph and adult). Negative number means the fold increased expression in adults (relation between adult and nymph)

saliva contains at least three proteins described as lipocalin. The presence of lipocalins in tick saliva is related to control of inflammatory processes and interference with host hemostatic functions [112-114]. Furthermore, the importance of lipocalins in nymph and adult saliva has been demonstrated, since the expression of lipocalins in 
insects and ticks is up-regulated in response to injury [115], as well as to viral [116] or bacterial infections [117], enhancing tick immune responses and resilience to infection.

\section{Detoxification/oxidation}

In nymph and adult saliva, various proteins involved in processes of detoxification and redox buffering were detected, including glutathione S-transferase, aldehyde dehydrogenase, sulfotransferase, and catalase. These proteins could detoxify oxidants generated during blood meal acquisition and/or host oxidants associated with inflammation. The antioxidant expression levels differ throughout arthropod development [118]. In H. longicornis saliva, the abundance of antioxidant proteins is higher in adults, when compared to nymphs (Tables 1, 2 and 3). A glutathione peroxidase was the only protein of this class identified in nymph saliva. This protein has been well characterized for its antioxidant and antiinflammatory activity in mammals [119], and could be related as an immunomodulatory protein from $H$. longicornis saliva. Sulfotransferase may inactivate dopamine, the secretagogue found in the salivary gland of ticks [120]. Glutathione S-transferase expression has been associated with resistance to acaricides and insecticides in many species [121, 122]. In addition, it has been proposed that GST secreted by parasites has immunomodulatory activity due to the alteration of the cytokine gene expression profile, modulation of immune cell proliferation, and decrease in oxidative ability of phagocytes $[123,124]$. The roles of GST and other proteins in the detoxification of endogenous toxin preventing and repairing the damage of ROS generated by hemoglobin degradation has been described [125], and the induction of the expression of these proteins in response to oxidative stress have been observed, supporting the antioxidant physiological role [126, 127]. Furthermore, immunization experiments showed the potential use of tick GST to protect hosts against tick infestation $[50,51]$.

\section{Cytoskeletal}

Proteins in H. longicornis saliva associated with cytoskeletal and structural cellular function were identified, including actin, tubulin, paramyosin, among others, which are fundamental to intracellular transport and cellular division. Notably, these proteins were abundantly expressed in adult saliva, when compared to nymph saliva, suggesting that the presence of such proteins has a physiological explanation, as opposed to tick/host tissue contamination during saliva collection (Table 4). Since these are intracellular proteins, and considering that most lack signal peptides, it may be hypothesized that they are released as consequence of damage, degeneration, or apoptosis of salivary gland acines [128, 129]. Furthermore, the presence of apocrine secretion in tick salivary gland is described [130]. Another structural protein has been identified in $R$. microplus saliva is paramyosin. This protein is secreted in saliva and recognized during the tick infestation, further suggesting that it may possess additional, non-muscle functions in the tick-host relationship [131]. Troponin Ilike molecule was detected in $H$. longicornis saliva, with angiogenesis inhibitor activity impairing host tissue repair and helping the tick feeding process [132]. These observations suggested that these proteins could have roles other than structural functions.

\section{Metabolism}

A wide variety of enzymes and proteins related to carbohydrate, lipid and amino acid metabolism and to the energetic pathways was observed in $H$. longicornis saliva. This finding is in accordance with other tick saliva proteomes. Similarly to cytoskeletal proteins, proteins that belong to this class are predominant in adult tick saliva (Table 1, 2 and 3). Although functional activity of these proteins remains unknown concerning tick feeding, the activity of some proteins related to metabolism has been characterized.

A salivary enolase from O. moubata was described acting as a plasminogen receptor, and may play a role stimulating host fibrinolysis and maintaining blood fluidity during tick feeding [133]. Similar activity was described for enolases from other parasites [134, 135].

Another group of metabolism-related proteins with a possible role in tick feeding is formed by chitinases. Chitinases are either active or inactive, based on the functional domain. Active chitinases are mostly described as responsible for the hydrolytic cleavage of the $\beta$-glycosidic linkages between GlcNAc residues of chitin, involved in molting and growth of arthropods [136]. On the other hand, inactive chitinases were suggested to be involved in the maintenance of a stable feeding site and in the activity of a potential immunoglobulin $\mathrm{G}$ binding protein in $A$. americanum [137]. It is interesting to note that we show the presence of a putative inactive chitinase only in nymphs (Table 1). However, previous studies showed the presence of an inactive chitinase in adults, as a secreted saliva protein $[16,138]$.

Enzymes of the o phospholipase A2 family play important roles in phospholipid digestion, rearrangement of cellular membrane phospholipid structures, inflammatory responses, defense and predation mechanisms, and signal transduction [139]. These enzymatic activities have been identified in tick saliva, and are speculated to stimulate tick prostaglandin E2 production [140]. Tick saliva proteomic studies have identified these proteins [16], pointing to their presence in tick saliva. A phospholipase A2 was identified exclusively in nymph saliva (Table 1). These proteins could provide the anti- 
inflammatory, anti-hemostatic, and vasodilator activity required for long-term blood feeding.

It should be noted that these metabolism-related proteins have been identified in tick saliva, and may be classified as moonlighting proteins, so they can have other distinct functions [141, 142]. Several studies on moonlighting proteins are being carried out, and the discovery of new functions could afford deeper insights into metabolism-related proteins in tick feeding physiology.

\section{Differential expression between nymph and adult female saliva proteins}

Ixodid ticks begin attachment by cutting into the host skin, followed by secretion of cement, a process that may take from one to two days. When completely affixed to the wound site, these ticks feed slowly from the pooled blood formed, for several days [143-145]. During feeding, the salivary gland of adult ixodid ticks undergoes remarkable growth and differentiation, which is accompanied by significant increases in the rate of proteins synthesis $[46,146]$. It has been proposed that different tick feeding conditions might affect salivary gland transcription of hard ticks. This feature is related to different vertebrate host exposure and distinct developmental stages, leading to changes in salivary transcription dynamics, as shown previously [14, 16, 32, 99, 147-150].

As shown in other tick saliva proteomes [14, 16], the protein content of tick saliva in different developmental stages varies, such as in this case from nymph to adult female ticks in $H$. longicornis. Of the 135 proteins detected in $H$. longicornis saliva, 30 proteins were identified in nymph saliva, 74 proteins were identified in fully engorged adult females, and 31 were detected in both stages (Fig. 2, Tables 1, 2 and 3). Nymph-specific proteins are represented mostly by proteinase inhibitors and a secreted conserved proteins group (Fig. 3 and Table 1), while adult specific proteins are represented primarily by proteins related to energy metabolism, oxidant/detoxification metabolism, and cytoskeleton (Fig. 3 and Table 2). This finding in adult saliva may be related to salivary gland degeneration starting after adult ticks detach from the host [128, 151].

Changes in expression levels were observed for 11 of the 31 identified tick proteins detected in both stages. The changes in the protein secretion were determined by pairwise comparison between nymphs and fully engorged H. longicornis female tick saliva using the TFold module from PatternLab for Proteomics platform [37], and were shown to be statistically significant (Fig. 4 and Table 4). The range in fold change was shown to be greater in the specific-tick proteins than in host protein secreted in nymph saliva. The most significantly affected nymph upregulated proteins were a hemelipoprotein (up-regulated 34.69-fold), a serpin (up-regulated 8.99-fold), a ribosomal protein s27a (up-regulated 7.98-fold), a cystatin (upregulated 5.47 -fold), and a glutathione peroxidase (upregulated 5.46-fold). The physiological meaning of these differences is not clear. However, as discussed previously, hemelipoproteins are known by their heme-binding function [63], and expression in early developmental stages of tick feeding observed here is in accordance with that found in $R$. microplus [16]. Proteinase inhibitors were described as early stage secreted saliva proteins in tick, with anticoagulant and immunomodulatory properties, modulating host defense systems trigged against tick feeding $[14,16,75,76,78,87]$. This observation can be related to the nature of the blood feeding process, throughout the different stages of tick as they have to use unique proteins to counteract host defenses, especially for fully engorged nymphs, which need to molt into adults and prepare to feed on another host.

\section{Host proteins}

As shown previously for other tick species $[13,15,16$, 30, 31], a large number of host proteins were identified both in nymph and adult $H$. longicornis saliva (Table 1 , 2 and 3). It was demonstrated that ticks transport intact proteins across the digestive system to the hemolymph. After blood ingestion, host blood proteins such as albumin and immunoglobulin cross the midgut epithelium of ticks, and are detected in tick tissues, including secretion into saliva $[15,152,153]$. This finding suggests that the presence of host proteins in tick saliva may be a real and common recycling system present in ticks, not a result of contamination during saliva collection.

In $H$. longicornis saliva, 100 proteins matched the rabbit database. Host identified proteins in tick saliva included proteins related to (i) immunity, such as immunoglobulins, complement system proteins, and antimicrobial proteins; (ii) heme/iron metabolism-related proteins, like serum albumin, peroxiredoxin, serotransferrin, apolipoprotein, and hemopexin; (iii) proteinase inhibitors of the serpin and alpha-2-macroglobulin superfamilies; and (iv) hemoglobin/red blood cells-related products. A set of 13 rabbit proteins was found only in nymph saliva samples, mostly rabbit keratin (Table 4, Fig. 3, and Additional file 3: Figure S1), suggesting that host keratin from tick mouthparts reached saliva during collection, since cleaning of host tissue in nymphs' mouthparts is more critical than in adults, due to its smaller size. Forty-eight rabbit proteins were only found in this adult saliva, and 30 rabbit proteins were present in both samples (Fig. 2).

The presence of different classes of host proteins in the saliva of the two tick developmental stages suggests the existence of a selective uptake process for host proteins (Fig. 3 and Additional file 3: Figure S1, Tables 1, 2, and 3 ) as observed in other studies $[15,16]$. Furthermore, 
the relationship between concentrations of these proteins in saliva is different from that observed in host blood. This data is similar to findings observed in other tick species $[30,31,153,154]$. An explanation for the presence of host proteins in tick saliva is that ticks recycle pivotal host proteins in order to subvert their role in the host and/or using host proteins in specific tick physiologic systems. Utilization of host hemoglobin as substrate to generate antimicrobial peptides against microorganisms was observed in R. microplus [155] and O. moubata [156]. As observed in $R$. microplus, here we found the same profile for proteins related to heme/iron metabolism. While the major tick heme-binding protein is secreted into saliva from nymphs, its expression decreases in adults. Reduction of heme-binding proteins in adults was accompanied by an increase in the host heme-binding proteins serum albumin, hemopexin, apolipoprotein, and peroxiredoxin (Fig. 3, Table 1, 2 and 3). These observations could suggest that the tick replaces hemelipoproteins by host derived heme-binding proteins, since hemelipoproteins are used for vitellogenesis at the end of the feeding process [62]. The host-derived transferrin was described in the hemolymph of $D$. variabilis and in whole nymphal ticks of $A$. americanum [157, 158]. Recently, a study showed the movement of host-derived transferrin $H$. longicornis, particularly from the midgut to the ovary, via hemolymph [159]. In the same way, $R$. microplus re-use heme from blood meal to synthetize heme proteins during protein synthesis [160].

Mammalian serpins are described endogenous regulators of host defenses against tick feeding [161, 162]. Host proteins of the serpin superfamily were identified in saliva, including alpha-1-antiprotease, antithrombin III, and alpha-2-antiplasmin. These proteins regulate enzymes such as neutrophil elastase, thrombin, and plasmin. It is important to find out whether these host proteins have the potential to inhibit their own serine proteinases. The presence of immunoglobulin chains could be explained as a tick self-defense system, since antibodies remain in an active form in tick hemolymph [152].

These observations suggest that the use of host proteins in tick physiology is not an unusual occurrence, and that these proteins may have an important physiology role in tick feeding process.

\section{Conclusions}

H. longicornis tick saliva has not been previously studied due to the considerable difficulty to collect saliva. Previously, researchers used salivary gland extract instead of saliva for protein analysis. This study describes the first proteome analysis of saliva of nymph and adult $H$. longicornis. Despite the use of saliva of fully engorged ticks, we could identify several tick proteins that can provide useful information for basic and applied aspects of the hostparasite interaction. The role of saliva proteins in the contact between a tick and the host is crucial during feeding process, and the knowledge about salivary components may improve the understanding of tick physiology, aiding the identification of a new target for tick control.

\section{Additional files}

Additional file 1: Table S1. Tick proteins in H. longicornis saliva identified by mass spectrometry. Name, classification based on function and/or protein family and annotation and accession numbers of best match identities obtained using BLASTP against the several protein databases are provided in the accompanying spreadsheet (Hyperlinked Excel spreadsheet, zipped).

Additional file 2: Table S2. Host proteins in $\mathrm{H}$. longicornis saliva identified by mass spectrometry. Name, classification based in function and/or protein family and annotation and accession numbers of best match identities obtained using BLASTP against the several protein databases are provided in the accompanying spreadsheet (Hyperlinked Excel spreadsheet, zipped).

Additional file 3: Figure S1. Functional classification of Haemaphysalis longicornis salivary proteins identified in fully engorged nymph (nymph) and fully engorged adult females (adult). Host proteins detected in nymph ( $A$ and $C$ ) and adult ( $B$ and $D$ ). Proteins divided as detected both in nymph (C) and adult (D) or detected exclusively in nymph (A) or adult (B) and classified in groups according to their function and/or protein family. Pie charts represent the percentage of proteins found in each group with respect to normalized spectral counting for each sample.

\section{Competing interests}

The authors declare that they have no competing interests.

\section{Authors' contributions}

LT, MSI, MY, and ISV designed the study. LT, MSI, JKD, and AFMP carried out the experiments. LT, MSI, TK, JKD, JRY, AFMP, AM, MY, and ISV analyzed the data. LT, MSI, TK, AFMP, AM, MY, and ISV drafted the manuscript. All authors read and approved the final manuscript.

\section{Authors' information}

Lucas Tirloni and Mohammad Saiful Islam are co-first authors. Myung-Jo You and Itabajara da Silva Vaz Jr are co-senior authors.

\section{Acknowledgments}

This project was supported by FAPERJ, INCT-Entomologia Molecular, CNPq and CAPES from Brazil; National Center for Research Resources (5P41RR011823), National Institute of General Medical Sciences (8P41GM103533) from USA, and research fund of Chonbuk National University in 2015 from Korea. Authors would like to thank Dr. José M. C. Ribeiro for providing the VB programs used in protein annotation.

\section{Author details}

${ }^{1}$ Centro de Biotecnologia, Universidade Federal do Rio Grande do Sul, Porto Alegre, RS, Brazil. ²Department of Veterinary Parasitology, College of Veterinary Medicine and Bio-safety Research Centre, Chonbuk National University, Jeonju, Republic of Korea. ${ }^{3}$ Department of Medicine, Surgery and Obstetrics, Faculty of Veterinary and Animal Science, Hajee Mohammad Danesh Science and Technology University, Dinajpur, Bangladesh.

${ }^{4}$ Department of Chemical Physiology, The Scripps Research Institute, La Jolla, CA, USA. ${ }^{5}$ Centro de Pesquisas em Biologia Molecular e Funcional, Instituto Nacional de Ciência e Tecnologia em Tuberculose (INCT-TB), Pontifícia Universidade Católica do Rio Grande do Sul (PUCRS), Porto Alegre, RS, Brazil. ${ }^{6}$ Department of Veterinary Pathobiology, College of Veterinary Medicine, Texas A\&M University, College Station, TX, USA. ${ }^{7}$ Faculdade de Veterinária, Universidade Federal do Rio Grande do Sul, Porto Alegre, RS, Brazil. 
Received: 29 March 2015 Accepted: 27 May 2015

\section{Published online: 24 June 2015}

\section{References}

1. Sun J, Liu Q, Lu L, Ding G, Guo J, Fu G, et al. Coinfection with four genera of bacteria (Borrelia, Bartonella, Anaplasma, and Ehrlichia) in Haemaphysalis longicornis and Ixodes sinensis ticks from China. Vector Borne Zoonotic Dis. 2008;8:791-5.

2. Lee MJ, Chae JS. Molecular detection of Ehrlichia chaffeensis and Anaplasma bovis in the salivary glands from Haemaphysalis longicornis ticks. Vector Borne Zoonotic Dis. 2010;10:411-3.

3. Zou Y, Wang Q, Fu Z, Liu P, Jin H, Yang H, et al. Detection of spotted fever group Rickettsia in Haemaphysalis longicornis from Hebei Province, China. J Parasitol. 2011;97:960-2.

4. Lee JH, Park HS, Jang WJ, Koh SE, Park TK, Kang SS, et al. Identification of the Coxiella sp. detected from Haemaphysalis longicornis ticks in Korea. Microbiol Immunol. 2004:48:125-30

5. Lee JH, Park HS, Jung KD, Jang WJ, Koh SE, Kang SS, et al. Identification of the spotted fever group rickettsiae detected from Haemaphysalis longicornis in Korea. Microbiol Immunol. 2003;47:301-4.

6. Guan G, Moreau E, Liu J, Hao X, Ma M, Luo J, et al. Babesia sp. BQ1 (Lintan): molecular evidence of experimental transmission to sheep by Haemaphysalis qinghaiensis and Haemaphysalis longicornis. Parasitol Int. 2010;59:265-7.

7. Li Y, Luo J, Guan G, Ma M, Liu A, Liu J, et al. Experimental transmission of Theileria uilenbergi infective for small ruminants by Haemaphysalis longicornis and Haemaphysalis qinghaiensis. Parasitol Res. 2009;104:1227-31.

8. Hoogstraal H, Roberts FH, Kohls GM, Tipton VJ. Review of Haemaphysalis (kaiseriana) longicornis Neumann (resurrected) of Australia, New Zealand, New Caledonia, Fiji, Japan, Korea, and Northeastern China and USSR, and its parthenogenetic and bisexual populations (Ixodoidea, Ixodidae). J Parasitol. 1968:54:1197-213.

9. Francischetti IM, Sa-Nunes A, Mans BJ, Santos IM, Ribeiro JM. The role of saliva in tick feeding. Front Biosci (Landmark Ed). 2009;14:2051-88.

10. Mans BJ. Evolution of vertebrate hemostatic and inflammatory control mechanisms in blood-feeding arthropods. J Innate Immun. 2011;3:41-51.

11. Ribeiro JM. Blood-feeding arthropods: live syringes or invertebrate pharmacologists? Infect Agents Dis. 1995;4:143-52.

12. Ribeiro JM, Francischetti IM. Role of arthropod saliva in blood feeding: sialome and post-sialome perspectives. Annu Rev Entomol. 2003;48:73-88.

13. Diaz-Martin V, Manzano-Roman R, Valero L, Oleaga A, Encinas-Grandes A, Perez-Sanchez R. An insight into the proteome of the saliva of the argasid tick Ornithodoros moubata reveals important differences in saliva protein composition between the sexes. J Proteomics. 2013;80C:216-35.

14. Mudenda L, Pierle SA, Turse JE, Scoles GA, Purvine SO, Nicora CD, et al. Proteomics informed by transcriptomics identifies novel secreted proteins in Dermacentor andersoni saliva. Int J Parasitol. 2014;44:1029-37.

15. Oliveira CJ, Anatriello E, de Miranda-Santos IK, Francischetti IM, Sa-Nunes A, Ferreira BR, et al. Proteome of Rhipicephalus sanguineus tick saliva induced by the secretagogues pilocarpine and dopamine. Ticks Tick Borne Dis. 2013;4(6):469-77.

16. Tirloni L, Reck J, Terra RM, Martins JR, Mulenga A, Sherman NE, et al. Proteomic analysis of cattle tick Rhipicephalus (Boophilus) microplus saliva: a comparison between partially and fully engorged females. PLoS One. 2014:9:e94831.

17. Carvalho-Costa T, Mendes M, Da SM, Da CT, Tiburcio M, Anhe A, et al. Immunosuppressive effects of Amblyomma cajennense tick saliva on murine bone marrow-derived dendritic cells. Parasit Vectors. 2015;8:22.

18. Maritz-Olivier C, Stutzer C, Jongejan F, Neitz AW, Gaspar AR. Tick antihemostatics: targets for future vaccines and therapeutics. Trends Parasitol. 2007:23:397-407.

19. Oliveira CJ, Sa-Nunes A, Francischetti IM, Carregaro V, Anatriello E, Silva JS, et al. Deconstructing tick saliva: non-protein molecules with potent immunomodulatory properties. J Biol Chem. 2011;286:10960-9.

20. Steen NA, Barker SC, Alewood PF. Proteins in the saliva of the Ixodida (ticks): pharmacological features and biological significance. Toxicon. 2006:47:1-20.

21. Nuttall PA, Labuda M. Tick-host interactions: saliva-activated transmission. Parasitology. 2004;129(Suppl):S177-89.

22. Parizi LF, Githaka NW, Logullo C, Konnai S, Masuda A, Ohashi K, et al. The quest for a universal vaccine against ticks: cross-immunity insights. Vet J. 2012;194:158-65.
23. Imamura S, Konnai S, Vaz IS, Yamada S, Nakajima C, Ito Y, et al. Effects of anti-tick cocktail vaccine against Rhipicephalus appendiculatus. Jpn J Vet Res. 2008;56:85-98.

24. Havlikova S, Roller L, Koci J, Trimnell AR, Kazimirova M, Klempa B, et al. Functional role of 64P, the candidate transmission-blocking vaccine antigen from the tick, Rhipicephalus appendiculatus. Int J Parasitol. 2009;39:1485-94.

25. Labuda M, Trimnell AR, Lickova M, Kazimirova M, Davies GM, Lissina O, et al. An antivector vaccine protects against a lethal vector-borne pathogen. PLoS Pathog. 2006;2:e27.

26. Neelakanta G, Sultana H. Transmission-blocking vaccines: focus on antivector vaccines against tick-borne diseases. Arch Immunol Ther Exp (Warsz). 2014;63(3):169-79.

27. Ciprandi A, de Oliveira SK, Masuda A, Horn F, Termignoni C. Boophilus microplus: its saliva contains microphilin, a small thrombin inhibitor. Exp Parasitol. 2006;114:40-6.

28. Horn F, Dos Santos PC, Termignoni C. Boophilus microplus anticoagulant protein: an antithrombin inhibitor isolated from the cattle tick saliva. Arch Biochem Biophys. 2000;384:68-73.

29. Liao M, Zhou J, Gong H, Boldbaatar D, Shirafuji R, Battur B, et al. Hemalin, a thrombin inhibitor isolated from a midgut CDNA library from the hard tick Haemaphysalis longicornis. J Insect Physiol. 2009:55:164-73.

30. Madden RD, Sauer JR, Dillwith JW. A proteomics approach to characterizing tick salivary secretions. Exp Appl Acarol. 2002;28:77-87.

31. Valenzuela JG, Francischetti IM, Pham VM, Garfield MK, Mather TN, Ribeiro JM. Exploring the sialome of the tick Ixodes scapularis. J Exp Biol. 2002;205:2843-64.

32. Radulovic ZM, Kim TK, Porter LM, Sze SH, Lewis L, Mulenga A. A 24-48 h fed Amblyomma americanum tick saliva immuno-proteome. BMC Genomics. 2014:15:518.

33. Lewis LA, Radulovic Z, Kim TK, Porter L, Mulenga A. Identification of $24 \mathrm{~h}$ Ixodes scapularis immunogenic tick saliva proteins. Ticks Tick Borne Dis. 2015;6(3):424-34.

34. McDonald WH, Tabb DL, Sadygov RG, MacCoss MJ, Venable J, Graumann J, et al. MS1, MS2, and SQT-three unified, compact, and easily parsed file formats for the storage of shotgun proteomic spectra and identifications. Rapid Commun Mass Spectrom. 2004;18:2162-8.

35. Xu T, Venable JD, Park SK, Conciorva D, Lu B, Liao L, et al. ProLuCID, a fast and sensitive tandem mass spectra-based protein identification program. Mol Cell Proteomics. 2006;5:S174.

36. Carvalho PC, Fischer JS, XU T, Cociorva D, Balbuena TS, Valente RH, et al. Search engine processor: filtering and organizing peptide spectrum matches. Proteomics. 2012;12:944-9.

37. Carvalho PC, Fischer JS, Xu T, Yates JR, III, Barbosa VC. PatternLab: from mass spectra to label-free differential shotgun proteomics. Curr Protoc Bioinformatics. 2012:40:13.19:13.19.1-13.19.18.

38. Zybailov B, Mosley AL, Sardiu ME, Coleman MK, Florens L, Washburn MP. Statistical analysis of membrane proteome expression changes in Saccharomyces cerevisiae. J Proteome Res. 2006;5:2339-47.

39. Lewis S, Ashburner M, Reese MG. Annotating eukaryote genomes. Curr Opin Struct Biol. 2000;10:349-54.

40. Marchler-Bauer A, Lu S, Anderson JB, Chitsaz F, Derbyshire MK, DeWeeseScott C, et al. CDD: a Conserved Domain Database for the functional annotation of proteins. Nucleic Acids Res. 2011;39:D225-9.

41. Tatusov RL, Fedorova ND, Jackson JD, Jacobs AR, Kiryutin B, Koonin EV, et al. The COG database: an updated version includes eukaryotes. BMC Bioinformatics. 2003:4:41.

42. Bateman A, Birney E, Cerruti L, Durbin R, Etwiller L, Eddy SR, et al. The Pfam protein families database. Nucleic Acids Res. 2002;30:276-80.

43. Schultz J, Copley RR, Doerks T, Ponting CP, Bork P. SMART: a web-based tool for the study of genetically mobile domains. Nucleic Acids Res. 2000;28:231-4.

44. Karim S, Singh P, Ribeiro JM. A deep insight into the sialotranscriptome of the gulf coast tick, Amblyomma maculatum. PLoS One. 2011;6:e28525.

45. Yu Z, Wang H, Wang T, Sun W, Yang X, Liu J. Tick-borne pathogens and the vector potential of ticks in China. Parasit Vectors. 2015;8:24.

46. Binnington KC. Sequential changes in salivary gland structure during attachment and feeding of the cattle tick, Boophilus microplus. Int J Parasitol. 1978;8:97-115.

47. Kaufman $W$. The influence of various factors on fluid secretion by in vitro salivary glands of ixodid Ticks. J Exp Biol. 1976;64:727-42.

48. McSwain JL, Essenberg RC, Sauer JR. Oral secretion elicited by effectors of signal transduction pathways in the salivary glands of Amblyomma americanum (Acari: Ixodidae). J Med Entomol. 1992;29:41-8. 
49. Sauer JR, McSwain JL, Bowman AS, Essenberg RC. Tick salivary gland physiology. Annu Rev Entomol. 1995;40:245-67.

50. Parizi LF, Utiumi KU, Imamura S, Onuma M, Ohashi K, Masuda A, et al. Cross immunity with Haemaphysalis longicornis glutathione S-transferase reduces an experimental Rhipicephalus (Boophilus) microplus infestation. Exp Parasitol. 2011;127:113-8.

51. Parizi LF, Reck Jr J, Oldiges DP, Guizzo MG, Seixas A, Logullo C, et al. Multiantigenic vaccine against the cattle tick Rhipicephalus (Boophilus) microplus: a field evaluation. Vaccine. 2012;30:6912-7.

52. Kotsyfakis M, Anderson JM, Andersen JF, Calvo E, Francischetti IM, Mather TN, et al. Cutting edge: Immunity against a "silent" salivary antigen of the Lyme vector Ixodes scapularis impairs its ability to feed. J Immunol. 2008;181:5209-12.

53. Galay RL, Miyata T, Umemiya-Shirafuji R, Maeda H, Kusakisako K, Tsuji N, et al. Evaluation and comparison of the potential of two ferritins as anti-tick vaccines against Haemaphysalis longicornis. Parasit Vectors. 2014;7:482.

54. Hajdusek O, Almazan C, Loosova G, Villar M, Canales M, Grubhoffer L, et al. Characterization of ferritin 2 for the control of tick infestations. Vaccine. 2010;28:2993-8.

55. Imamura S, da Silva VJ I, Sugino M, Ohashi K, Onuma M. A serine protease inhibitor (serpin) from Haemaphysalis longicornis as an anti-tick vaccine. Vaccine. 2005;23:1301-11.

56. Imamura S, Namangala B, Tajima T, Tembo ME, Yasuda J, Ohashi K, et al. Two serine protease inhibitors (serpins) that induce a bovine protective immune response against Rhipicephalus appendiculatus ticks. Vaccine. 2006;24:2230-7.

57. Jittapalapong S, Kaewhom P, Pumhom P, Canales M, de la Fuente J, Stich RW. Immunization of rabbits with recombinant serine protease inhibitor reduces the performance of adult female Rhipicephalus microplus. Transbound Emerg Dis. 2010;57:103-6.

58. Prevot PP, Couvreur B, Denis V, Brossard M, Vanhamme L, Godfroid E. Protective immunity against Ixodes ricinus induced by a salivary serpin. Vaccine. 2007;25:3284-92.

59. Sugino M, Imamura S, Mulenga A, Nakajima M, Tsuda A, Ohashi K, et al. A serine proteinase inhibitor (serpin) from ixodid tick Haemaphysalis longicornis; cloning and preliminary assessment of its suitability as a candidate for a tick vaccine. Vaccine. 2003;21:2844-51.

60. Taheri M, Nabian S, Ranjbar M, Mazaheri NR, Gerami SA, Sazmand A. Study of vitellogenin in Boophilus annulatus tick larvae and its immunological aspects. Trop Biomed. 2014;31:398-405.

61. Tellam RL, Kemp D, Riding G, Briscoe S, Smith D, Sharp P, et al. Reduced oviposition of Boophilus microplus feeding on sheep vaccinated with vitellin. Vet Parasitol. 2002;103:141-56.

62. Logullo C, Moraes J, Dansa-Petretski M, Vaz IS, Masuda A, Sorgine MH, et al. Binding and storage of heme by vitellin from the cattle tick, Boophilus microplus. Insect Biochem Mol Biol. 2002;32:1805-11.

63. Maya-Monteiro CM, Daffre S, Logullo C, Lara FA, Alves EW, Capurro ML, et al. HeLp, a heme lipoprotein from the hemolymph of the cattle tick, Boophilus microplus. J Biol Chem. 2000;275:36584-9.

64. Dutra FF, Alves LS, Rodrigues D, Fernandez PL, de Oliveira RB, Golenbock DT, et al. Hemolysis-induced lethality involves inflammasome activation by heme. Proc Natl Acad Sci U S A. 2014;111:E4110-8.

65. Dutra FF, Bozza MT. Heme on innate immunity and inflammation. Front Pharmacol. 2014;5:115.

66. Graca-Souza AV, Arruda MA, de Freitas MS, Barja-Fidalgo C, Oliveira PL. Neutrophil activation by heme: implications for inflammatory processes. Blood. 2002;99:4160-5.

67. Pichu S, Ribeiro JM, Mather TN, Francischetti IM. Purification of a serine protease and evidence for a protein $C$ activator from the saliva of the tick, Ixodes scapularis. Toxicon. 2014;77:32-9.

68. Franta Z, Frantova H, Konvickova J, Horn M, Sojka D, Mares M, et al. Dynamics of digestive proteolytic system during blood feeding of the hard tick Ixodes ricinus. Parasit Vectors. 2010;3:119.

69. Sojka D, Franta Z, Horn M, Caffrey CR, Mares M, Kopacek P. New insights into the machinery of blood digestion by ticks. Trends Parasitol. 2013;29:276-85.

70. Tsuji N, Miyoshi T, Battsetseg B, Matsuo T, Xuan X, Fujisaki K. A cysteine protease is critical for Babesia spp. transmission in Haemaphysalis ticks. PLoS Pathog. 2008;4:e1000062.

71. Julius D, Basbaum Al. Molecular mechanisms of nociception. Nature. 2001;413:203-10

72. Ribeiro JM, Mather TN. Ixodes scapularis: salivary kininase activity is a metallo dipeptidyl carboxypeptidase. Exp Parasitol. 1998;89:213-21.
73. Gettins PG. Serpin structure, mechanism, and function. Chem Rev. 2002;102:4751-804.

74. Mulenga A, Khumthong R, Blandon RA. Molecular and expression analysis of a family of the Amblyomma americanum tick Lospins. J Exp Biol. 2007;210:3188-98.

75. Ibelli AM, Kim TK, Hill CC, Lewis LA, Bakshi M, Miller S, et al. A blood mealinduced Ixodes scapularis tick saliva serpin inhibits trypsin and thrombin, and interferes with platelet aggregation and blood clotting. Int J Parasitol. 2014:44:369-79.

76. Mulenga A, Kim T, Ibelli AM. Amblyomma americanum tick saliva serine protease inhibitor 6 is a cross-class inhibitor of serine proteases and papainlike cysteine proteases that delays plasma clotting and inhibits platelet aggregation. Insect Mol Biol. 2013;22:306-19.

77. Prevot PP, Adam B, Boudjeltia KZ, Brossard M, Lins L, Cauchie P, et al. Antihemostatic effects of a serpin from the saliva of the tick Ixodes ricinus. J Biol Chem. 2006;281:26361-9.

78. Chmelar J, Oliveira CJ, Rezacova P, Francischetti IM, Kovarova Z, Pejler G, et al. A tick salivary protein targets cathepsin $\mathrm{G}$ and chymase and inhibits host inflammation and platelet aggregation. Blood. 2011;117:736-44.

79. Leboulle G, Crippa M, Decrem Y, Mejri N, Brossard M, Bollen A, et al. Characterization of a novel salivary immunosuppressive protein from Ixodes ricinus ticks. J Biol Chem. 2002;277:10083-9.

80. Prevot PP, Beschin A, Lins L, Beaufays J, Grosjean A, Bruys L, et al. Exosites mediate the anti-inflammatory effects of a multifunctional serpin from the saliva of the tick Ixodes ricinus. FEBS J. 2009;276:3235-46.

81. Chisenhall DM, Christofferson RC, McCracken MK, Johnson AM, LondonoRenteria B, Mores CN. Infection with dengue-2 virus alters proteins in naturally expectorated saliva of Aedes aegypti mosquitoes. Parasit Vectors. 2014;7:252.

82. Williams AR, Zakutansky SE, Miura K, Dicks MD, Churcher TS, Jewell KE, et al. Immunisation against a serine protease inhibitor reduces intensity of Plasmodium berghei infection in mosquitoes. Int J Parasitol. 2013;43:869-74.

83. Abrahamson M, Alvarez-Fernandez M, Nathanson CM. Cystatins. Biochem Soc Symp. 2003;70:179-99.

84. Kotsyfakis M, Sa-Nunes A, Francischetti IM, Mather TN, Andersen JF, Ribeiro JM. Antiinflammatory and immunosuppressive activity of sialostatin L, a salivary cystatin from the tick Ixodes scapularis. J Biol Chem. 2006;281:26298-307.

85. Kotsyfakis M, Karim S, Andersen JF, Mather TN, Ribeiro JM. Selective cysteine protease inhibition contributes to blood-feeding success of the tick Ixodes scapularis. J Biol Chem. 2007;282:29256-63.

86. Parizi LF, Sabadin GA, Alzugaray MF, Seixas A, Logullo C, Konnai S, et al. Rhipicephalusmicroplus and Ixodesovatus cystatins in tick blood digestion and evasion of host immune response. Parasit Vectors. 2015;8:122

87. Sa-Nunes A, Bafica A, Antonelli LR, Choi EY, Francischetti IM, Andersen JF, et al. The immunomodulatory action of sialostatin $L$ on dendritic cells reveals its potential to interfere with autoimmunity. J Immunol. 2009;182:7422-9.

88. Karim S, Miller NJ, Valenzuela J, Sauer JR, Mather TN. RNAi-mediated gene silencing to assess the role of synaptobrevin and cystatin in tick blood feeding. Biochem Biophys Res Commun. 2005;334:1336-42.

89. Kotsyfakis M, Horka H, Salat J, Andersen JF. The crystal structures of two salivary cystatins from the tick Ixodes scapularis and the effect of these inhibitors on the establishment of Borrelia burgdorferi infection in a murine model. Mol Microbiol. 2010;77:456-70.

90. Fogaca AC, Almeida IC, Eberlin MN, Tanaka AS, Bulet P, Daffre S. Ixodidin, a novel antimicrobial peptide from the hemocytes of the cattle tick Boophilus microplus with inhibitory activity against serine proteinases. Peptides. 2006;27:667-74.

91. Sasaki SD, Cotrin SS, Carmona AK, Tanaka AS. An unexpected inhibitory activity of Kunitz-type serine proteinase inhibitor derived from Boophilus microplus trypsin inhibitor on cathepsin L. Biochem Biophys Res Commun. 2006;341:266-72.

92. Cvirn G, Gallistl S, Koestenberger M, Kutschera J, Leschnik B, Muntean W. Alpha 2-macroglobulin enhances prothrombin activation and thrombin potential by inhibiting the anticoagulant protein C/protein S system in cord and adult plasma. Thromb Res. 2002;105:433-9.

93. Harpel PC. Human plasma alpha 2-macroglobulin: an inhibitor of plasma kallikrein. J Exp Med. 1970;132:329-52.

94. Meijers JC, Tijburg PN, Bouma BN. Inhibition of human blood coagulation factor Xa by alpha 2-macroglobulin. Biochemistry. 1987;26:5932-7.

95. Banks RE, Evans SW, Van LF, Alexander D, McMahon MJ, Whicher JT. Measurement of the 'fast' or complexed form of alpha 2 macroglobulin in 
biological fluids using a sandwich enzyme immunoassay. J Immunol Methods. 1990;126:13-20.

96. Bonacci GR, Caceres LC, Sanchez MC, Chiabrando GA. Activated alpha(2)macroglobulin induces cell proliferation and mitogen-activated protein kinase activation by LRP-1 in the $J 774$ macrophage-derived cell line. Arch Biochem Biophys. 2007;460:100-6.

97. Anatriello E, Ribeiro JM, de Miranda-Santos IK, Brandao LG, Anderson JM, Valenzuela JG, et al. An insight into the sialotranscriptome of the brown dog tick, Rhipicephalus sanguineus. BMC Genomics. 2010;11:450.

98. Gibson AK, Smith Z, Fuqua C, Clay K, Colbourne JK. Why so many unknown genes? Partitioning orphans from a representative transcriptome of the lone star tick Amblyomma americanum. BMC Genomics. 2013;14:135.

99. Mulenga A, Blandon M, Khumthong R. The molecular basis of the Amblyomma americanum tick attachment phase. Exp Appl Acarol. 2007;41:267-87.

100. Mulenga A, Kim TK, Ibelli AM. Deorphanization and target validation of cross-tick species conserved novel Amblyomma americanum tick saliva protein. Int J Parasitol. 2013;43:439-51.

101. Xu XL, Cheng TY, Yang H, Yan F, Yang Y. De Novo Sequencing, Assembly and Analysis of Salivary Gland Transcriptome of Haemaphysalis flava and Identification of Sialoprotein Genes. Infect Genet Evol. 2015;32:135-42.

102. Megraw T, Kaufman TC, Kovalick GE. Sequence and expression of Drosophila Antigen 5-related 2, a new member of the CAP gene family. Gene. 1998;222:297-304.

103. Garcia GR, Gardinassi LG, Ribeiro JM, Anatriello E, Ferreira BR, Moreira HN, et al. The sialotranscriptome of Amblyomma triste, Amblyomma parvum and Amblyomma cajennense ticks, uncovered by 454-based RNA-seq. Parasit Vectors. 2014;7:430.

104. Anderson JM, Oliveira F, Kamhawi S, Mans BJ, Reynoso D, Seitz AE, et al. Comparative salivary gland transcriptomics of sandfly vectors of visceral leishmaniasis. BMC Genomics. 2006:7:52.

105. Bishop R, Lambson B, Wells C, Pandit P, Osaso J, Nkonge C, et al. A cement protein of the tick Rhipicephalus appendiculatus, located in the secretory e cell granules of the type III salivary gland acini, induces strong antibody responses in cattle. Int J Parasitol. 2002;32:833-42.

106. Zhou J, Gong H, Zhou Y, Xuan X, Fujisaki K. Identification of a glycinerich protein from the tick Rhipicephalus haemaphysaloides and evaluation of its vaccine potential against tick feeding. Parasitol Res. 2006;100:77-84

107. Maruyama SR, Anatriello E, Anderson JM, Ribeiro JM, Brandao LG, Valenzuela $J G$, et al. The expression of genes coding for distinct types of glycine-rich proteins varies according to the biology of three metastriate ticks, Rhipicephalus (Boophilus) microplus, Rhipicephalus sanguineus and Amblyomma cajennense. BMC Genomics. 2010;11:363.

108. Liu XY, Bonnet SI. Hard tick factors implicated in pathogen transmission. PLoS Negl Trop Dis. 2014;8:e2566.

109. Mans BJ, Neitz AW. Exon-intron structure of outlier tick lipocalins indicate a monophyletic origin within the larger lipocalin family. Insect Biochem Mol Biol. 2004;34:585-94.

110. Paesen GC, Adams PL, Harlos K, Nuttall PA, Stuart DI. Tick histamine-binding proteins: isolation, cloning, and three-dimensional structure. Mol Cell. 1999;3:661-71.

111. Ganfornina MD, Kayser H, Sanchez D. Lipocalins in Arthropoda: diversification and functional explorations. In: Madame Curie Bioscinece Database. [Internet]. Austin (TX). Edited by Lands Bioscience: Lands Bioscience; 2000. Available from: http://www.ncbi.nlm.nih.gov/books/NBK6576/.

112. Beaufays J, Adam B, Menten-Dedoyart C, Fievez L, Grosjean A, Decrem Y, et al. Ir-LBP, an Ixodes ricinus tick salivary LTB4-binding lipocalin, interferes with host neutrophil function. PLoS One. 2008:3:e3987.

113. Mans BJ, Ribeiro JM. Function, mechanism and evolution of the moubatinclade of soft tick lipocalins. Insect Biochem Mol Biol. 2008;38:841-52.

114. Mans BJ, Ribeiro JM, Andersen JF. Structure, function, and evolution of biogenic amine-binding proteins in soft ticks. J Biol Chem. 2008;283:18721-33.

115. Kim HJ, Je HJ, Cheon HM, Kong SY, Han J, Yun CY, et al. Accumulation of $23 \mathrm{kDa}$ lipocalin during brain development and injury in Hyphantria cunea. Insect Biochem Mol Biol. 2005;35:1133-41.

116. McNally KL, Mitzel DN, Anderson JM, Ribeiro JM, Valenzuela JG, Myers TG, et al. Differential salivary gland transcript expression profile in Ixodes scapularis nymphs upon feeding or flavivirus infection. Ticks Tick Borne Dis. 2012;3:18-26.

117. Cheng PH, Mans BJ, Neitz AW, Gaspar AR. Savicalin, a lipocalin from hemocytes of the soft tick, Ornithodoros savignyi. Exp Appl Acarol. 2010;52:313-26
118. Heekin AM, Guerrero FD, Bendele KG, Saldivar L, Scoles GA, Dowd SE, et al. The ovarian transcriptome of the cattle tick, Rhipicephalus (Boophilus) microplus, feeding upon a bovine host infected with Babesia bovis. Parasit Vectors. 2013;6:276.

119. Chu FF, Esworthy RS, Doroshow JH. Role of Se-dependent glutathione peroxidases in gastrointestinal inflammation and cancer. Free Radic Biol Med. 2004;36:1481-95.

120. Pichu S, Yalcin EB, Ribeiro JM, King RS, Mather TN. Molecular characterization of novel sulfotransferases from the tick, Ixodes scapularis. BMC Biochem. 2011;12:32.

121. Coles TB, Dryden MW. Insecticide/acaricide resistance in fleas and ticks infesting dogs and cats. Parasit Vectors. 2014;7:8.

122. da Silva Jr VI, Torino LT, Michelon A, Sanchez Ferreira CA, de Freitas DR J, Termignoni $\mathrm{C}$, et al. Effect of acaricides on the activity of a Boophilus microplus glutathione S-transferase. Vet Parasitol. 2004;119:237-45.

123. Ouaissi A, Ouaissi M, Sereno D. Glutathione S-transferases and related proteins from pathogenic human parasites behave as immunomodulatory factors. Immunol Lett. 2002;81:159-64

124. Plumas-Marty B, Verwaerde C, Loyens M, Velge P, Taibi A, Cesbron MF, et al. Trypanosoma cruzi glutathione-binding proteins: immunogenicity during human and experimental Chagas' disease. Parasitology. 1992;104(Pt 1):87-98.

125. Toh SQ, Glanfield A, Gobert GN, Jones MK. Heme and blood-feeding parasites: friends or foes? Parasit Vectors. 2010;3:108.

126. Freitas DR, Rosa RM, Moraes J, Campos E, Logullo C, da Silva Jr VI, et al. Relationship between glutathione S-transferase, catalase, oxygen consumption, lipid peroxidation and oxidative stress in eggs and larvae of Boophilus microplus (Acarina: Ixodidae). Comp Biochem Physiol A Mol Integr Physiol. 2007;146:688-94

127. Wei SH, Clark AG, Syvanen M. Identification and cloning of a key insecticidemetabolizing glutathione S-transferase (MdGST-6A) from a hyper insecticide-resistant strain of the housefly Musca domestica. Insect Biochem Mol Biol. 2001;31:1145-53.

128. Freitas DR, Rosa RM, Moura DJ, Seitz AL, Colodel EM, Driemeier D, et al. Cell death during preoviposition period in Boophilus microplus tick. Vet Parasitol. 2007; 144:321-7.

129. Furquim KC, Bechara GH, Camargo Mathias MI. Degeneration of salivary glands of males of the tick Rhipicephalus sanguineus (Latreille, 1806) (Acari, |xodidae). Vet Parasitol. 2008;154:325-35.

130. Coons LB, Roshdy MA. Fine structure of the salivary glands of unfed male Dermacentor variabilis (Say) (Ixodoidea: Ixodidae). J Parasitol. 1973;59:900-12.

131. Leal BF, Seixas A, Mattos RT, Coutinho ML, Masuda A, da Silva Jr Vl, et al. Tissue expression and the host's immunological recognition of a Rhipicephalus microplus paramyosin. Vet Parasitol. 2013;197:304-11.

132. Fukumoto S, Sakaguchi T, You M, Xuan X, Fujisaki K. Tick troponin I-like molecule is a potent inhibitor for angiogenesis. Microvasc Res. 2006;71:218-21.

133. Diaz-Martin V, Manzano-Roman R, Oleaga A, Encinas-Grandes A, PerezSanchez R. Cloning and characterization of a plasminogen-binding enolase from the saliva of the argasid tick Ornithodoros moubata. Vet Parasitol, 2013;191:301-14.

134. Jolodar A, Fischer P, Bergmann S, Buttner DW, Hammerschmidt S, Brattig NW. Molecular cloning of an alpha-enolase from the human filarial parasite Onchocerca volvulus that binds human plasminogen. Biochim Biophys Acta. 2003;1627:111-20

135. Vanegas G, Quinones W, Carrasco-Lopez C, Concepcion JL, Albericio F, Avilan L. Enolase as a plasminogen binding protein in Leishmania mexicana. Parasitol Res. 2007:101:1511-6.

136. Hamid R, Khan MA, Ahmad M, Ahmad MM, Abdin MZ, Musarrat J, et al. Chitinases: an update. J Pharm Bioallied Sci. 2013;5:21-9.

137. Kim TK, Curran J, Mulenga A. Dual silencing of long and short Amblyomma americanum acidic chitinase forms weakens the tick cement cone stability. J Exp Biol. 2014;217:3493-503.

138. Kotsyfakis M, Schwarz A, Erhart J, Ribeiro JM. Tissue- and time-dependent transcription in Ixodes ricinus salivary glands and midguts when blood feeding on the vertebrate host. Sci Rep. 2015;5:9103.

139. Burke JE, Dennis EA. Phospholipase A2 structure/function, mechanism, and signaling. J Lipid Res. 2009;50(Suppl):S237-42.

140. Bowman AS, Gengler CL, Surdick MR, Zhu K, Essenberg RC, Sauer JR, et al. A novel phospholipase A2 activity in saliva of the lone star tick, Amblyomma americanum (L.). Exp Parasitol. 1997;87:121-32.

141. Huberts DH, van der Klei IJ. Moonlighting proteins: an intriguing mode of multitasking. Biochim Biophys Acta. 2010;1803:520-5. 
142. Mani M, Chen C, Amblee V, Liu H, Mathur T, Zwicke G, et al. MoonProt: a database for proteins that are known to moonlight. Nucleic Acids Res. 2015;43:D277-82.

143. Anderson JF, Magnarelli LA. Biology of ticks. Infect Dis Clin North Am. 2008;22:195-215.

144. Ribeiro JM. Role of saliva in blood-feeding by arthropods. Annu Rev Entomol. 1987:32:463-78.

145. Ribeiro JM. How ticks make a living. Parasitol Today. 1995;11:91-3.

146. McSwain JL, Essenberg RC, Sauer JR. Protein changes in the salivary glands of the female lone star tick, Amblyomma americanum, during feeding. J Parasitol. 1982;68:100-6.

147. Schwarz A, von Reumont BM, Erhart J, Chagas AC, Ribeiro JM, Kotsyfakis M. De novo Ixodes ricinus salivary gland transcriptome analysis using two nextgeneration sequencing methodologies. FASEB J. 2013;27:4745-56.

148. Schwarz A, Tenzer S, Hackenberg M, Erhart J, Gerhold-Ay A, Mazur J, et al. A systems level analysis reveals transcriptomic and proteomic complexity in Ixodes ricinus midgut and salivary glands during early attachment and feeding. Mol Cell Proteomics. 2014;13:2725-35.

149. Chmelar J, Anderson JM, Mu J, Jochim RC, Valenzuela JG, Kopecky J. Insight into the sialome of the castor bean tickm, Ixodes ricinus. BMC Genomics. 2008:9:233.

150. Leboulle G, Rochez C, Louahed J, Ruti B, Brossard M, Bollen A, et al. Isolation of Ixodes ricinus salivary gland mRNA encoding factors induced during blood feeding. Am J Trop Med Hyg. 2002;66:225-33.

151. Bowman AS, Sauer JR. Tick salivary glands: function, physiology and future. Parasitology. 2004;129(Suppl):S67-81.

152. Vaz Jl, Martinez RH, Oliveira A, Heck A, Logullo C, Gonzales JC, et al. Functional bovine immunoglobulins in Boophilus microplus hemolymph. Vet Parasitol. 1996;62:155-60.

153. Wang $\mathrm{H}$, Nuttall PA. Excretion of host immunoglobulin in tick saliva and detection of lgG-binding proteins in tick haemolymph and salivary glands. Parasitology. 1994;109(Pt 4):525-30.

154. Francischetti IM, Anderson JM, Manoukis N, Pham VM, Ribeiro JM. An insight into the sialotranscriptome and proteome of the coarse bontlegged tick, Hyalomma marginatum rufipes. J Proteomics. 2011;74:2892-908.

155. Fogaca AC, da Silva PIJ, Miranda MT, Bianchi AG, Miranda A, Ribolla PE, et al. Antimicrobial activity of a bovine hemoglobin fragment in the tick Boophilus microplus. J Biol Chem. 1999;274:25330-4.

156. Nakajima Y, Ogihara K, Taylor D, Yamakawa M. Antibacterial hemoglobin fragments from the midgut of the soft tick, Ornithodoros moubata (Acari: Argasidae). J Med Entomol. 2003;40:78-81.

157. Ackerman S, Clare FB, McGill TW, Sonenshine DE. Passage of host serum components, including antibody, across the digestive tract of Dermacentor variabilis (Say). J Parasitol. 1981;67:737-40.

158. Wickramasekara S, Bunikis J, Wysocki V, Barbour AG. Identification of residual blood proteins in ticks by mass spectrometry proteomics. Emerg Infect Dis. 2008;14:1273-5.

159. Mori H, Galay RL, Maeda H, Matsuo T, Umemiya-Shirafuji R, Mochizuki M, et al. Host-derived transferrin is maintained and transferred from midgut to ovary in Haemaphysalis longicornis ticks. Ticks Tick Borne Dis. 2014;5:121-6.

160. Braz GR, Coelho HS, Masuda H, Oliveira PL. A missing metabolic pathway in the cattle tick Boophilus microplus. Curr Biol. 1999;9:703-6.

161. Mulenga A, Sugino M, Nakajim M, Sugimoto C, Onuma M. Tick-Encoded serine proteinase inhibitors (serpins); potential target antigens for tick vaccine development. J Vet Med Sci. 2001;63:1063-9.

162. Rau JC, Beaulieu LM, Huntington JA, Church FC. Serpins in thrombosis, hemostasis and fibrinolysis.J Thromb Haemost. 2007:5 Suppl 1:102-15.

\section{Submit your next manuscript to BioMed Central and take full advantage of:}

- Convenient online submission

- Thorough peer review

- No space constraints or color figure charges

- Immediate publication on acceptance

- Inclusion in PubMed, CAS, Scopus and Google Scholar

- Research which is freely available for redistribution 